\title{
$\mathbb{N} / \mathrm{IT}$
}

Karlsruher Institut für Technologie

\section{Modellgestützte Bewertung des Kraft-Wärme-Kopplungsgesetzes 2016 anhand ausgewählter Anwendungsfälle in Wohngebäuden}

Erik Merkel, Robert Kunze, Russell McKenna, Wolf Fichtner

No. 15 | Juni 2016

\section{WORKING PAPER SERIES IN PRODUCTION AND ENERGY}

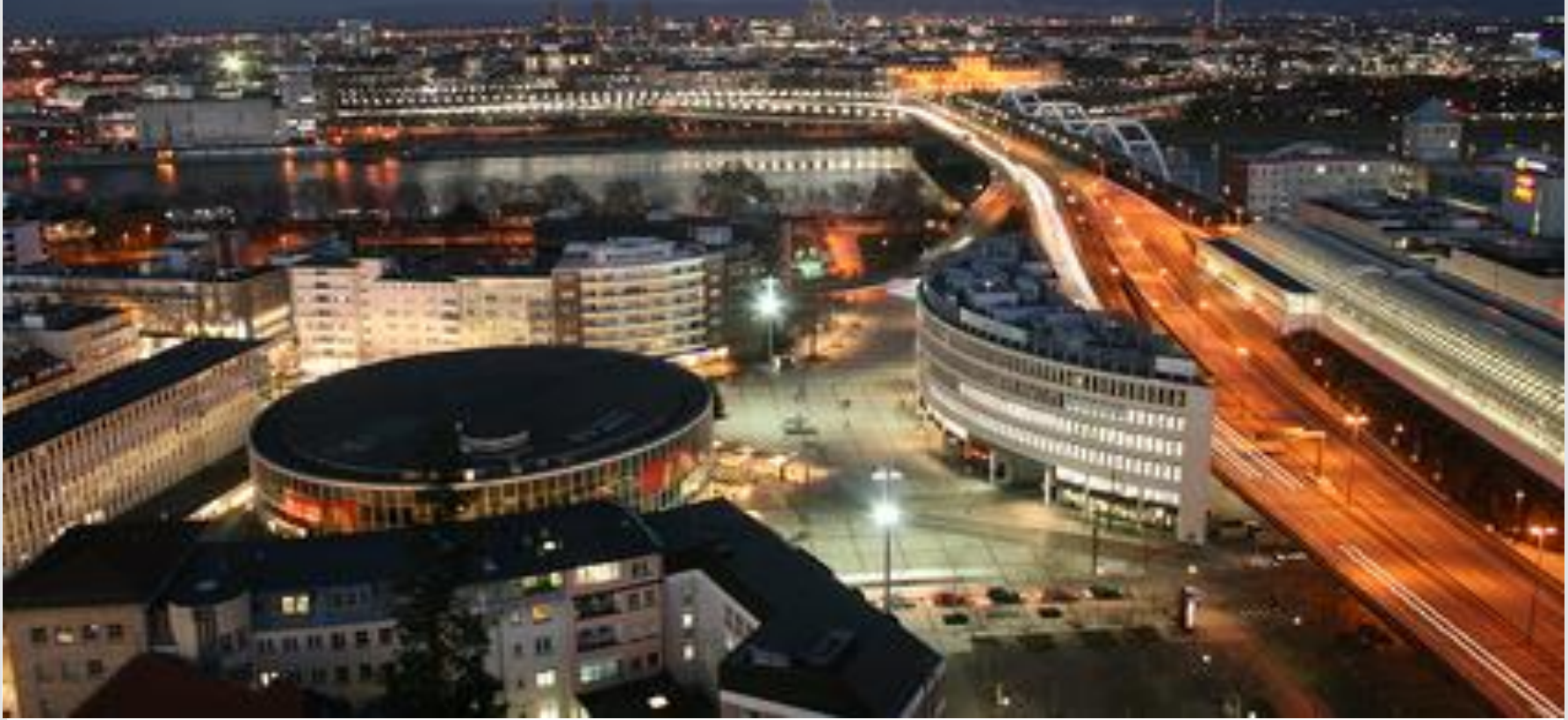




\title{
Modellgestützte Bewertung des Kraft-Wärme- Kopplungsgesetzes 2016 anhand ausgewählter Anwendungsfälle in Wohngebäuden
}

\author{
Erik Merkel, Robert Kunze, Russell McKenna, Wolf Fichtner
}

\begin{abstract}
Chair of Energy Economics, Institute for Industrial Production (IIP) at the Karlsruhe Institute of Technology (KIT), Hertzstr. 16, building 06.33, 76187 Karlsruhe, Germany

Tel.: +49 7216084 4582, email: mckenna@kit.edu
\end{abstract}

Der Kraft-Wärme-Kopplung wird auf Grund ihrer Energieeffizienz, breiten Anwendungsmöglichkeit in den Nachfragesektoren sowie Erzeugungsflexibilität eine Schlüsselrolle in der Umsetzung der Energiewende zugeschrieben. Die Energiepolitik der Bundesregierung in Deutschland sieht daher eine bedeutende Steigerung des Anteils dieser Technologie an der Elektrizitätserzeugung in zukünftigen Jahren vor. Als Instrument zur staatlichen Förderung des Ausbaus existiert zur Zielerreichung unter anderem das Kraft-Wärme-Kopplungsgesetz (KWKG) seit 2002. Zuletzt wurde dieses 2016 umfassend überarbeitet und enthält gewichtige Änderungen zur Vorgängerfassung, unter anderem zur Höhe und Dauer der Zuschlagzahlungen. Ziel dieses Beitrages ist die Bewertung dieser Veränderungen hinsichtlich technischer, wirtschaftlicher und ökologischer Aspekte mithilfe eines modellgestützten Ansatzes für ausgewählte Fallbeispiele zur Elektrizitäts- und Wärmeversorgung von Wohngebäuden für unterschiedliche räumliche Aggregationsebenen vom Einzelgebäude bis hin zum Quartier. Im Ergebnis zeigt sich für das Bezugsjahr 2016 die ökonomische Vorteilhaftigkeit des KWK-Einsatzes unter den Förderbedingungen des KWKG 2016 gegenüber des KWKG 2002 (zuletzt 2012 novelliert) für die Anwendungsfälle der mittleren betrachteten Aggregationsebenen (Straßenzüge und Häuserblöcke). Dagegen ist eine Umkehr der Wirtschaftlichkeit im Vergleich zu den Rahmenbedingungen der Vorgängerfassung für höhere Aggregationsebenen (Siedlung, Quartier) festzustellen. Bei letzteren kommt es insbesondere zu einer vergleichsweise kleineren Auslegung der Anlagen bei gleichzeitig höherer Vollbenutzungsstundenanzahl. Des Weiteren werden in diesem Beitrag Implikationen der KWKG-Neufassung von 2016 hinsichtlich technischer und ökologischer Aspekte aufgezeigt. 


\title{
Modellgestützte Bewertung des Kraft-Wärme-Kopplungsgesetzes 2016 anhand ausgewählter Anwendungsfälle in Wohngebäuden
}

\author{
Erik Merkel, Robert Kunze, Russell McKenna, Wolf Fichtner \\ Institut für Industriebetriebslehre und Industrielle Produktion (IIP), Lehrstuhl für Energiewirtschaft, Karlsruher Institut \\ für Technologie (KIT), Hertzstraße 16, 76187 Karlsruhe, Deutschland \\ erik.merkel@kit.edu, Tel. +49 721 608-44639, Fax. +49 721 608-44682
}

\section{Zusammenfassung}

Der Kraft-Wärme-Kopplung wird auf Grund ihrer Energieeffizienz, breiten Anwendungsmöglichkeit in den Nachfragesektoren sowie Erzeugungsflexibilität eine Schlüsselrolle in der Umsetzung der Energiewende zugeschrieben. Die Energiepolitik der Bundesregierung in Deutschland sieht daher eine bedeutende Steigerung des Anteils dieser Technologie an der Elektrizitätserzeugung in zukünftigen Jahren vor. Als Instrument zur staatlichen Förderung des Ausbaus existiert zur Zielerreichung unter anderem das Kraft-Wärme-Kopplungsgesetz (KWKG) seit 2002. Zuletzt wurde dieses 2016 umfassend überarbeitet und enthält gewichtige Änderungen zur Vorgängerfassung, unter anderem zur Höhe und Dauer der Zuschlagzahlungen. Ziel dieses Beitrages ist die Bewertung dieser Veränderungen hinsichtlich technischer, wirtschaftlicher und ökologischer Aspekte mithilfe eines modellgestützten Ansatzes für ausgewählte Fallbeispiele zur Elektrizitäts- und Wärmeversorgung von Wohngebäuden für unterschiedliche räumliche Aggregationsebenen vom Einzelgebäude bis hin zum Quartier. Im Ergebnis zeigt sich für das Bezugsjahr 2016 die ökonomische Vorteilhaftigkeit des KWK-Einsatzes unter den Förderbedingungen des KWKG 2016 gegenüber des KWKG 2002 (zuletzt 2012 novelliert) für die Anwendungsfälle der mittleren betrachteten Aggregationsebenen (Straßenzüge und Häuserblöcke). Dagegen ist eine Umkehr der Wirtschaftlichkeit im Vergleich zu den Rahmenbedingungen der Vorgängerfassung für höhere Aggregationsebenen (Siedlung, Quartier) festzustellen. Bei letzteren kommt es insbesondere zu einer vergleichsweise kleineren Auslegung der Anlagen bei gleichzeitig höherer Vollbenutzungsstundenanzahl. Des Weiteren werden in diesem Beitrag Implikationen der KWKG-Neufassung von 2016 hinsichtlich technischer und ökologischer Aspekte aufgezeigt.

Schüsselwörter: Kraft-Wärme-Kopplung, KWKG 2016, Gemischt-ganzzahlige Programmierung

Model-based assessment of the implications of the new version of the Combined Heat and Power Act 2016 based on selected use cases

\begin{abstract}
Cogeneration is a promising technology in the energy transition amongst other things in view of its energy efficiency and its broad applicability in the household, tertiary and industry sectors. For the promotion of cogeneration technologies, support schemes are in place in German energy policy. Amongst other things the Combined Heat and Power (CHP) Act serves as the main instrument for the promotion since 2002. In the new version of the CHP Act of 2016 the German Federal Government strives towards a more market-oriented design resulting in significant amendments featuring inter alia a highly case-dependent tariff design with modified levels and durations of remuneration. It is therefore the aim of the present study to assess these modifications with respect to technical, economic and ecological aspects in a model-based approach by means of selected use cases at different levels of spatial aggregation for residential buildings. Results point to an economic advantageousness for
\end{abstract}


cases of the medium energy consumption level (streets and blocks of houses) under the new regime as well as the reversal of this attractiveness for objects of high consumption (neighbourhoods, districts). Particularly, CHP plants tend be dimensioned comparatively smaller with a greater number of full-load hours under the new regime. Furthermore, implications with regard to technical and ecological aspects are shown in this contribution.

\section{$1 \quad$ Einleitung}

Zum 1. Januar 2016 trat die Neufassung des Kraft-Wärme-Kopplungsgesetzes (KWKG) in Kraft, welche zahlreiche Änderungen gegenüber der zuletzt 2012 novellierten Vorgängerfassung von 2002 (im Folgenden als KWKG 2012 bezeichnet) beinhaltet. Weitreichend überarbeitet wurden insbesondere die Förderregelungen für KWK-Technologien, welche sich maßgeblich auf die Wirtschaftlichkeit des Anlageneinsatzes auswirken. Diesbezüglich wurden im Vorfeld die Entwürfe zum Gesetz von verschiedenen Interessenverbänden teilweise sehr kritisch beurteilt (ASUE et al. 2015). Die geäußerte Kritik fand in der im Dezember 2015 vom Deutschen Bundestag verabschiedeten KWKG-Neufassung nur vereinzelt Berücksichtigung.

Auch mit dem KWKG 2016 beabsichtigt die Bundesregierung, die KWK-Nutzung als einen wichtigen Baustein zum Gelingen der Energiewende zu unterstützen. Der maximale Gesamtbetrag für Förderungen über das KWKG wurde mit der Neufassung auf 1,5 Mrd. € pro Jahr verdoppelt. Allerdings verdeutlicht das Gesetz nun stärker die Vorrangstellung der Elektrizitätserzeugung aus Erneuerbaren Energien gegenüber der gekoppelten ElektrizitätsWärme-Erzeugung aus konventionellen Energieträgern. Letztere wird ausschließlich über das KWKG gefördert. So wurde zum einen die bisherige Zielsetzung des 25-prozentigen Anteils von KWK-Strom an der gesamten Nettostromerzeugung auf absolute Zielwerte beschränkt: Bis 2020 sollen 110 TWh und bis $2025120 \mathrm{TWh}$ Nettostrom aus KWK-Anlagen bereitgestellt werden. 2014 betrug die KWK-Nettostromerzeugung 101,6 TWh. Dies entspricht einem Anteil von ca. 17,3\% an den 589 TWh insgesamt erzeugten Nettostrom in diesem Jahr (BMWi 2016). Zum anderen entfallen nach dem KWKG 2016 die Zuschlagzahlungen für erzeugten KWK-Strom in den Zeiträumen, in denen die Stundenkontraktwerte an der EPEX Spot SE in Paris für die Preiszone Deutschland/Österreich gleich Null oder negativ sind. Die wesentliche Ursache für solche Preise ist im Zusammenspiel einer verstärkten Einspeisung aus PV- und Windkraftanlagen bei gleichzeitig geringer Nachfrage und einer zu geringen Flexibilität der regelbaren Kraftwerke zu sehen.

Mit dem KWKG 2016 soll bei der Förderung die wirtschaftliche Vorteilhaftigkeit des Anlagenbetriebs stärker Berücksichtigung finden und gleichzeitig die Integration der Anlagen in den Elektrizitätsmarkt vorangetrieben werden. Vor diesem Hintergrund weisen die Regelungen der KWKG-Neufassung gewisse Parallelen zum Erneuerbare Energien-Gesetz (EEG) auf, bei dem dahin gehende Anpassungen bereits in den Novellierungen von 2012 und 2014 umgesetzt wurden. Dazu zählt zunächst die Einführung einer verpflichtenden Direktvermarktung für KWK-Anlagen ab $100 \mathrm{~kW}_{\text {el }}$ elektrischer Leistung. Ebenso stellt die vorgenommene Differenzierung der Förderhöhe für den in öffentliche Versorgungsnetze eingespeisten und für die nicht eingespeiste Elektrizität eine entsprechende Neuerung im KWKG dar. Die Einspeisung wird nun deutlich höher bezuschlagt. Letzteres wurde als notwendig erachtet, da sich die Erwirtschaftung der erforderlichen Deckungsbeiträge für KWK-Anlagen mit vorrangiger Netzeinspeisung aufgrund der niedrigen Stromgroßhandelspreise in den letzten Jahren als zunehmend schwieriger erwies. Der Markt setzte somit auch kaum Anreize für Neuinvestitionen in diesem Bereich. In diesem Zusammenhang ist auch die zusätzlich in den Anwendungsbereich des KWKG aufgenommene und bis 2020 beschränkte Bezuschlagung der Stromeinspeisung aus bestehenden Gas-KWK-Anlagen größer $2 \mathrm{MW}_{\mathrm{el}}$ zu sehen, 
welche nicht nach EEG und nicht (mehr) nach dem KWKG gefördert werden. Die Förderhöhe für nicht eingespeisten KWK-Strom wurde dagegen herabgesetzt und deutlich beschränkt. Die Bezuschlagung erfolgt nur noch für KWK-Strom aus Anlagen bis maximal $100 \mathrm{~kW}_{\mathrm{el}}$ oder den Eigenverbrauch in stromintensiven Industrien sowie für nicht eingespeisten Strom, sofern hierfür die volle EEG-Umlage entrichtet wird.

Eine weitere wesentliche Neuerung bildet die Einschränkung des KWKG-Anwendungsbereiches hinsichtlich des eingesetzten Energieträgers. Erfolgte die Förderung bis dato brennstoffneutral, entfällt sie ab 2016 vollständig für (wieder) in Betrieb genommene Anlagen auf Basis von Kohle. Darüber hinaus wird für den ins öffentliche Versorgungsnetz eingespeisten Strom aus neuen, modernisierten oder nachgerüsteten Anlagen ein Bonuszuschlag gewährt, wenn diese Anlagen Kohle-KWK ersetzen. Eine zusammenfassende Gegenüberstellung wesentlicher Änderungen der Förderbedingungen auf Grundlage des KWKG 2016 im Vergleich zur letzten Novellierung der Vorgängerfassung des Gesetzes zeigt Tabelle 1.

Tab. 1 Gegenüberstellung wesentlicher Förderregelungen des KWKG 2012 und des KWKG 2016

\begin{tabular}{|c|c|c|c|c|c|}
\hline \multirow[t]{2}{*}{ Kriterium } & \multirow[t]{2}{*}{ Leistungsbereich } & \multicolumn{2}{|c|}{ KWKG 2012} & \multicolumn{2}{|c|}{ KWKG 2016} \\
\hline & & $\begin{array}{l}\text { Netzein- } \\
\text { speisung }\end{array}$ & Selbstnutzung & $\begin{array}{l}\text { Netzein- } \\
\text { speisung }\end{array}$ & $\begin{array}{l}\text { Selbstnut- } \\
\text { zung }\end{array}$ \\
\hline \multirow{5}{*}{ Zuschlaghöhe $\left(\mathrm{ct} / \mathrm{kWh}_{\mathrm{el}}\right)$} & $\mathrm{x} \leq 50 \mathrm{~kW}_{\mathrm{el}}$ & 5,41 & 5,41 & 8,00 & 4,00 \\
\hline & $50<\mathrm{x} \leq 100 \mathrm{~kW}_{\mathrm{el}}$ & 4,00 & 4,00 & 6,00 & 3,00 \\
\hline & $100<\mathrm{x} \leq 250 \mathrm{~kW}_{\mathrm{el}}$ & 4,00 & 4,00 & 5,00 & 0,00 \\
\hline & $250<\mathrm{x} \leq 2.000 \mathrm{~kW}_{\mathrm{el}}$ & 2,40 & 2,40 & 4,40 & 0,00 \\
\hline & $\mathrm{x} \geq 2.000 \mathrm{~kW}_{\mathrm{el}}$ & 1,80 & 1,80 & 3,10 & 0,00 \\
\hline \multirow{2}{*}{ Zuschlagdauer } & $\mathrm{x} \leq 50 \mathrm{~kW}_{\mathrm{el}}$ & \multicolumn{2}{|c|}{10 Jahre oder $30.000 \mathrm{VBSt}$} & \multicolumn{2}{|c|}{$60.000 \mathrm{VBSt}$} \\
\hline & $\mathrm{x}>50 \mathrm{~kW}_{\mathrm{el}}$ & \multicolumn{2}{|c|}{$30.000 \mathrm{VBSt}$} & \multicolumn{2}{|c|}{$30.000 \mathrm{VBSt}$} \\
\hline \multirow{2}{*}{$\begin{array}{l}\text { Vergütung von } \\
\text { netzeingespeistem } \\
\text { Strom zzgl. vermiedener } \\
\text { Netznutzungsentgelte } \\
\text { höherer Spannungsebenen }\end{array}$} & $\mathrm{x} \leq 100 \mathrm{~kW}_{\mathrm{el}}$ & \multirow{2}{*}{\multicolumn{2}{|c|}{$\begin{array}{l}\text { Direktvermarktung oder } \\
\text { kaufmännische Abnahme } \\
\text { (i. d. R. zum Preis für } \\
\text { Grundlaststrom des } \\
\text { vorangegangenen Quartals } \\
\text { an der European Energy } \\
\text { Exchange = üblicher Preis) }\end{array}$}} & \multicolumn{2}{|c|}{$\begin{array}{l}\text { Direktvermarktung oder } \\
\text { kaufmännische Abnahme } \\
\text { (üblicher Preis) }\end{array}$} \\
\hline & $\mathrm{x}>100 \mathrm{~kW}_{\mathrm{el}}$ & & & \multicolumn{2}{|c|}{ Direktvermarktungspflicht } \\
\hline $\begin{array}{l}\text { Marktbedingte Aussetzung } \\
\text { der Vergütungszahlung² }\end{array}$ & & \multicolumn{2}{|l|}{ Nein } & \multicolumn{2}{|l|}{$\mathrm{Ja}$} \\
\hline Pauschalierte Zahlung & $\mathrm{x} \leq 2 \mathrm{~kW}_{\mathrm{el}}$ & \multicolumn{2}{|c|}{$30.000 \mathrm{VBSt}$} & \multicolumn{2}{|c|}{$60.000 \mathrm{VBSt}$} \\
\hline $\begin{array}{l}\text { Zuschlagzahlungen für } \\
\text { Wärme- und Kältenetze }\end{array}$ & $\begin{array}{l}\text { Mittlerer } \\
\text { Nenndurchmesser } \leq \\
100 \mathrm{~mm}\end{array}$ & \multicolumn{2}{|c|}{$\begin{array}{l}100 € / \mathrm{m} \text { (höchstens } 40 \% \\
\text { der ansatzfähigen } \\
\text { Investition) }\end{array}$} & \multicolumn{2}{|c|}{$\begin{array}{l}100 € / \mathrm{m} \text { (höchstens } 40 \% \\
\text { der ansatzfähigen } \\
\text { Investition) }\end{array}$} \\
\hline
\end{tabular}




\begin{tabular}{|c|c|c|c|}
\hline & $\begin{array}{l}\text { Mittlerer } \\
\text { Nenndurchmesser > } \\
100 \mathrm{~mm}\end{array}$ & $\begin{array}{l}30 \% \text { der ansatzfähigen } \\
\text { Investition }\end{array}$ & $\begin{array}{l}30 \% \text { der ansatzfähigen } \\
\text { Investition }\end{array}$ \\
\hline \multirow{3}{*}{$\begin{array}{l}\text { Zuschlagzahlungen für } \\
\text { Wärme- und Kältespeicher }\end{array}$} & Volumen $\leq 50 \mathrm{~m}^{3}$ & $250 € / \mathrm{m}^{3}$ & $250 € / \mathrm{m}^{3}$ \\
\hline & Volumen $>50 \mathrm{~m}^{3}$ & $\begin{array}{l}30 \% \text { der ansatzfähigen } \\
\text { Investition }\end{array}$ & $\begin{array}{l}30 \% \text { der ansatzfähigen } \\
\text { Investition }\end{array}$ \\
\hline & $\begin{array}{l}\text { Höchstförderung pro } \\
\text { Projekt }\end{array}$ & 5 Mio. $€$ & 10 Mio. $€$ \\
\hline $\begin{array}{l}\text { Summe der } \\
\text { Zuschlagzahlungen (KWK- } \\
\text { Anlagen, Netze und } \\
\text { Speicher) }\end{array}$ & & 750 Mio. $€ / a$ & 1.500 Mio. $€ / \mathrm{a}$ \\
\hline
\end{tabular}

Das Ziel des vorliegenden Beitrags ist die Analyse der umfassenden Neuregelungen des KWKG 2016 im Hinblick auf die dadurch gesetzten Investitionsanreize für KWK-Anlagen. Der Fokus liegt dabei auf der gekoppelten Elektrizitäts-Wärme-Versorgung von Wohngebäuden. Hierfür bietet die modellgestützte Energiesystemanalyse geeignete Voraussetzungen für eine adäquate Beurteilung der rechtlichen Neuerungen. In dieser Untersuchung wird ein solcher Ansatz vorgestellt, welcher es gestattet, die Implikationen der unterschiedlichen KWK-Politiken aus einzelwirtschaftlicher Sicht und unter einem hohen Detaillierungsgrad technischer, wirtschaftlicher und ökologischer Aspekte für unterschiedliche Aggregationsebenen von Versorgungssystemen - vom Einzelgebäude bis hin zum Quartier - zu bewerten.

Der entwickelte Bewertungsansatz ist zur Beantwortung u. a. nachstehender Fragen geeignet: Wie ist ein ausgabenminimales dezentrales Wärmeversorgungssystem eines Nachfrageobjekts unter dem Vergütungsregime des KWKG 2012 bzw. KWKG 2016 beschaffen? Welches ist dabei die optimale Auslegung und Fahrweise der Anlagenkomponenten wie der KWK-Einheit und des Wärmespeichers? Welches sind die wirtschaftlichen, technischen und ökologischen Implikationen auch gegenüber einem Referenzversorgungssystem?

Der vorliegende Beitrag gliedert sich wie folgt: In Kapitel 2 wird die der Analyse zu Grunde liegende Methodik beschrieben und dabei ein Optimierungsmodell dezentraler Wärmeversorgungssysteme vorgestellt. Das dritte Kapitel weist zentrale Annahmen zur Datenbasis für die Modellrechnungen aus. Im vierten Kapitel werden die Ergebnisse der Modellläufe aufgezeigt und diskutiert. Eine Zusammenfassung sowie ein Ausblick auf zukünftige Untersuchungsvorhaben runden in Kapitel 5 den Beitrag ab. 


\section{Ein Modell zur Optimierung dezentraler Wärmeversorgungssysteme}

In diesem Kapitel wird der dieser Analyse zu Grunde liegende methodische Ansatz vorgestellt. Hierfür wird zunächst ein genereller Überblick über das methodische Vorgehen gegeben und anschließend auf ausgewählte zentrale Elemente des Modellierungsansatzes näher eingegangen.

\subsection{Methodisches Vorgehen}

Die Abbildung 1 stellt das methodische Vorgehen schematisch dar. Im Kern des Bewertungsansatzes befindet sich ein Optimierungsmodell dezentraler Wärmeversorgungssysteme, welches auf der gemischt-ganzzahligen linearen Programmierung beruht. Dieses ermittelt für gegebene Kombinationen von Gebäudeobjekten und Technologien unter der Prämisse minimaler Gesamtausgaben ein optimales Versorgungssystem für den Elektrizitäts- und Wärmeverbrauch. Als auswählbare und zu optimierende Technologien sind ein verbrennungsmotorisches Blockheizkraftwerk (BHKW), ein Spitzenlastkessel zur Wärmeversorgung sowie ein Wärmespeicher hinterlegt. Zusätzlich wird der Netzbezug von Elektrizität abgebildet. Im Modell wird neben einer Dimensionierung der Systemkomponenten des Versorgungssystems ebenso deren optimale Einsatzplanung auf der zeitlich hochaufgelösten Ebene bestimmt. Im Modell wird von einer Neu- bzw. Erstinstallation der Anlagen ausgegangen und daher eine bestehende Versorgung nicht berücksichtigt. Zur Abbildung der zeitlichen Veränderung der Parameter, welche insbesondere für die elektrische und thermische Nachfrage sowie die Elektrizitätsmarktdaten relevant sind, wird eine zeitliche Dekomposition im Modell verwirklicht. Dieser zufolge wird das kalendarische Jahr in neun repräsentative Wochen unterteilt, welche zu gleichem Anteil typische Wochen der Sommer-, Winterund Übergangsjahreszeit in 15-minütigen Zeitschritten abbilden. Der Betrachtungshorizont des Modells beträgt 20 Jahre. Dabei erfolgt die Verteilung der Nutzungsstunden gleichmäßig über den Planungszeitraum.

Das Optimierungsmodell ist in der algebraischen Modellierungssprache GAMS implementiert, die Datenhaltung der Ein- und Ausgabedateien ist in MS EXCEL verwirklicht. Für weiterführende Informationen zu dem Optimierungsmodell dezentraler Wärmeversorgungssysteme wird auf Merkel et al. (2015) verwiesen.

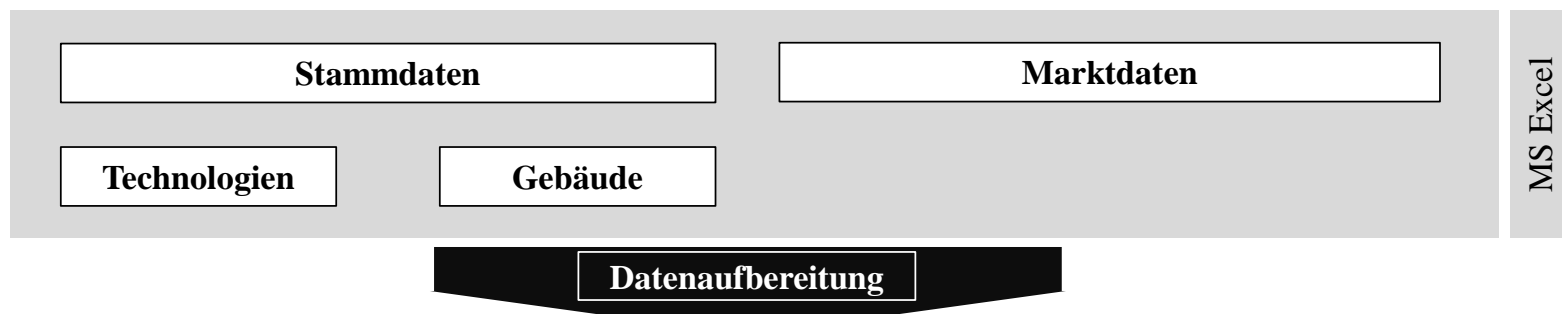

\section{Optimierungsmodell dezentraler Wärmeversorgungssysteme (MILP)}

Szenarioerstellung

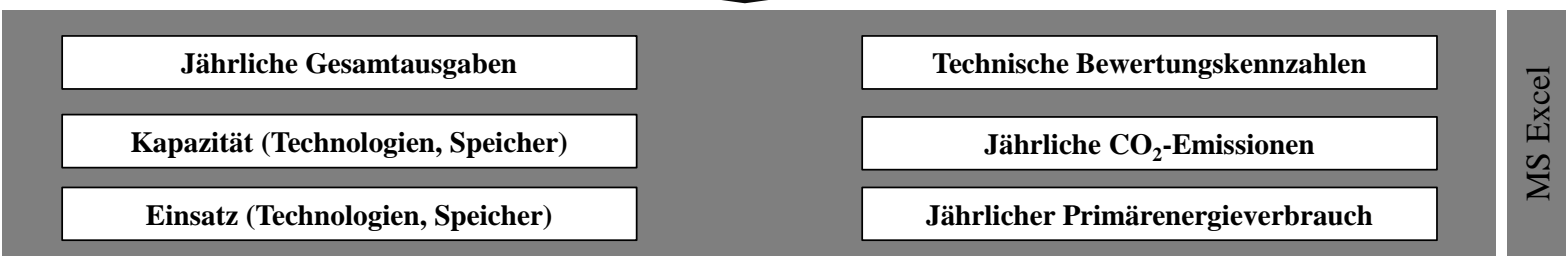

Abb. 1 Überblick über das methodische Vorgehen 
Weiterhin gehen verschiedene Größen in das Modell als Parameter ein. Diese betreffen zum einen die Stammdaten der Anlagen sowie der Gebäude und zum anderen als Marktdaten bezeichnete Preisinformationen des Elektrizitätsmarktes. Die Stammdaten sind technischer, wirtschaftlicher sowie ökologischer Natur und dienen der Charakterisierung sowohl der Anlagen als auch der Wohngebäude als Nachfrageobjekte im Optimierungsmodell. Als Marktdaten werden stündliche Spotmarktpreise für Elektrizität wie auch die vierteljährlichen durchschnittlichen Preise für Grundlaststrom an der EEX hinterlegt. Diese Daten entstammen in Anlehnung an Heffels et al. (2012) historischen Profilen.

Das Optimierungsmodell dezentraler Wärmeversorgungssysteme determiniert die jährlichen Gesamtausgaben für die Elektrizitäts- und Wärmeversorgung wie auch die Kapazität und den Einsatz der Erzeugungstechnologien und Speicher. Zudem werden technische Bewertungskennzahlen errechnet, welche sich auf den Energieautarkiegrad und die Eigenverbrauchsquote sowie die Netzinteraktion beziehen. Schließlich werden auch ökologische Ergebnisgrößen in Gestalt der jährlichen $\mathrm{CO}_{2}$-Emission sowie des jährlichen Primärenergieverbrauchs ausgewiesen.

Es existieren weiterhin drei Derivate des Optimierungsmodells: Eine Version, welche das Vergütungsregime nach dem KWKG 2012 abbildet, sowie eine Version, in der die Vergütungscharakteristika der KWKG-Neufassung aus dem Jahr 2016 hinterlegt sind. Zudem wird für den Referenzfall eine Modellversion erstellt, in der die Wärmeversorgung gänzlich über einen Niedertemperatur-Gaskessel und die Elektrizitätsversorgung über den Netzbezug sichergestellt wird. In den Modellversionen mit Einbezug von KWK-Anlagen kann jedoch ebenso nur ein Gaskessel sowie der ausschließliche Netzbezug von Elektrizität und damit der Referenzfall gewählt werden.

\subsection{Zielfunktion}

Die Gleichung 1 beschreibt die Zielfunktion des Optimierungsmodells. In diese finden sämtliche Zahlungen im Zusammenhang mit der Versorgung des Nachfrageobjekts mit Elektrizität und Wärme Eingang. Insbesondere werden kapital-, verbrauchs- und betriebsgebundene Ausgaben der KWK-Anlage, des Spitzenlastkessels und des Wärmespeichers berücksichtigt. Zusätzlich werden auch alle Zahlungen im Zusammenhang mit den Zuschlägen und Vergütungen nach dem KWKG einbezogen. Hierbei wird zwischen in das Versorgungsnetz eingespeister und selbstgenutzter Elektrizität unterschieden sowie die Vergütungshöhe als Funktion der installierten Kapazitätshöhe der KWK-Anlage abgebildet (vgl. 2) ${ }^{1}$.

$$
\begin{aligned}
& \min \mathrm{f}=\sum_{\mathrm{p}=1}^{\mathrm{P}}\left(\mathrm{c}_{\mathrm{inv}, \mathrm{p}}+\mathrm{c}_{\mathrm{fix}, \mathrm{p}}+\propto \cdot \sum_{\mathrm{t}^{\prime}=1}^{\mathrm{T}^{\prime}}\left(\mathrm{c}_{\mathrm{var}, \mathrm{p}, \mathrm{t}^{\prime}}-\mathrm{c}_{\text {rev }, \mathrm{p}, \mathrm{t}^{\prime}}\right)\right) \\
& \mathrm{c}_{\mathrm{rev}, \mathrm{p}, \mathrm{t}}=\mathrm{x}_{\mathrm{el}, \mathrm{e}}^{\mathrm{KWK}}(\mathrm{t}) \cdot \mathrm{c}_{\mathrm{rev}, \mathrm{e}}\left(\mathrm{x}_{\text {cap }}^{\mathrm{KWK}}\right)+\mathrm{x}_{\mathrm{el}, \mathrm{i}}^{\mathrm{KWK}}(\mathrm{t}) \cdot \mathrm{c}_{\text {rev }, \mathrm{i}}\left(\mathrm{x}_{\text {cap }}^{\mathrm{KWK}}\right) \forall \mathrm{t} \in \mathrm{T}
\end{aligned}
$$

Die kapitalgebundenen Ausgaben errechnen sich dabei als Produkt der Investition und des Annuitätenfaktors, in welchen der Zinssatz i sowie der Planungshorizont T eingehen. Mit Hilfe eines preisdynamischen Barwertfaktors wird überdies ermöglicht, in der Zielfunktion die Entwicklung der Bezugspreise für Erdgas und Elektrizität sowie der Großhandelspreise für Elektrizität über die Jahre abzubilden.

\footnotetext{
1 Im Modell wird die spezifische Vergütungshöhe zusätzlich nach Zugehörigkeit zur jährlichen unter dem KWKG geförderten Vollbenutzungsstundenanzahl und ferner nach Befreiung von der EEG-Umlage bei selbstgenutzter Elektrizität differenziert. Diese Sachverhalte sind in Gleichung 2 jedoch vereinfacht dargestellt.
} 


\subsection{Nebenbedingungen}

In dem Optimierungsmodell ist eine Vielzahl von Nebenbedingungen implementiert, welche darauf abstellen, technische, wirtschaftliche und ökologische Zusammenhänge des zu optimierenden Elektrizitäts- und Wärmeversorgungssystems abzubilden. Aus Übersichtlichkeitsgründen erfolgt an dieser Stelle nur eine qualitative Beschreibung dieser. Für einen umfassenden Überblick über die im Optimierungsmodell dezentraler Wärmeversorgungssysteme hinterlegten Nebenbedingungen sei auf Merkel et al. (2015) verwiesen. Im Modell finden sich u. a. Restriktionen zu minimalen und maximalen Leistungsgrenzen der Anlagen, zur Erfüllung der Nachfrage nach thermischer und elektrischer Energie, zu Energiebilanzgleichungen bei der Produktion von Elektrizität und Wärme und zu intertemporalen Bilanzgleichungen des Wärmespeichers. Weiterhin sind Gleichungen zur Bilanzierung der direkten und indirekten Emissionen von $\mathrm{CO}_{2}$ sowie des nicht-erneuerbaren und des gesamten Primärenergieaufwands hinterlegt. Zudem werden Größendegressionseffekte der Anlagen im Modell abgebildet, indem die konkave Kurve der Investition als Funktion der Kapazität stückweise linearisiert und mittels Variablen vom Typ SOS2 im Modell abgebildet wird. Für eine eingehende Darstellung der Implementierung wird dabei auf Merkel et al. (2015) verwiesen.

\subsection{Modellierung des Vergütungsregimes}

Eine wesentliche Eigenschaft der Modellierung besteht in der detaillierten Abbildung des Vergütungsregimes des KWKG. Dabei ist die Abhängigkeit der spezifischen Vergütungszahlungen in Höhe und Dauer von einer Reihe von Faktoren zu berücksichtigen. Diese beziehen sich auf die Anlagengröße (Kapazität), Nutzung der erzeugten Elektrizität in Form der Selbstnutzung bzw. der Netzeinspeisung sowie die Vermarktungsoption bei netzeingespeister Elektrizität unter dem KWKG 2016 in Gestalt der kaufmännischen Abnahme bzw. Direktvermarktungsoption (vgl. Kapitel 1).

Zur modelltechnischen Implementierung des Vergütungsregimes wird in dem Optimierungsmodell daher eine Unterscheidung der skizzierten Fälle mit Relevanz für die Vergütungshöhe und -dauer vorgenommen. Dies wird zum einen über die Differenzierung der kontinuierlichen Variablen für die Elektrizitätsflüsse der KWK-Anlage und zum anderen über die Einführung von Binärvariablen verwirklicht.

Demzufolge werden die Elektrizitätsflüsse der KWK-Anlage nach der Art der Nutzung, also Selbstnutzung und Netzeinspeisung, sowie jeweils nach Gestehungszeitpunkt innerhalb bzw. außerhalb der nach dem KWKG vergüteten Vollbenutzungsstundenanzahl unterschieden. Ferner erfolgt für die selbstgenutzte Elektrizität eine Unterscheidung der Entrichtungspflicht sowie der Befreiung von der anteiligen EEG-Umlage. Infolgedessen ergibt sich eine Gesamtzahl von 6 kontinuierlichen Variablen für die Elektrizitätsflüsse der KWK-Anlage je Zeitschritt. Dieses Vorgehen ermöglicht somit durch die Einführung von Nebenbedingungen die Begrenzung der Elektrizitätsflüsse und der dadurch induzierten Zuschlagzahlungen, welche innerhalb der nach dem KWKG bezuschlagten Vollbenutzungsstundenanzahl sowie unterhalb der Erzeugungshöhe der EEG-Umlage-Befreiung auftreten.

Die Binärvariablen dienen schließlich der Feststellung der Leistungsklasse sowie der Unterscheidung der Vermarktungsoption bei netzeingespeister Elektrizität unter den Rahmenbedingungen des KWKG 2016. Stellvertretend für die Vielzahl der benötigten Ungleichungen wird zur Überprüfung der Bedingung der KWKAnlagenkapazität kleiner bzw. größer $10 \mathrm{~kW}_{\mathrm{el}}$ zur Aufhebung der EEG-Umlage auf selbstverbrauchte Elektrizität eine Fallunterscheidung in den Ungleichungen 3 bis 5 vorgenommen. 
$\mathrm{x}_{\mathrm{cap}}^{\mathrm{KWK}} \leq 10 \cdot \mathrm{y}_{K W K, 1}^{E E G}+\mathrm{M} \cdot \mathrm{y}_{K W K, 2}^{E E G}$

$\mathrm{x}_{\text {cap }}^{\mathrm{KWK}} \geq(10+\varepsilon) \cdot \mathrm{y}_{K W K, 2}^{E E G}$

$\mathrm{y}_{K W K, 1}^{E E G}+\mathrm{y}_{K W K, 2}^{E E G} \leq 1$

Die Binärvariablen ermöglichen durch „Aktivierung“ bzw. „Deaktivierung“ somit die entsprechende Auswahl der im Modell als Parameter hinterlegten spezifischen Einzahlung für den jeweiligen Elektrizitätsfluss der KWKAnlage. Ferner kommen Binärvariablen in der Restriktion der Vergütungsdauer zum Tragen. Diese hängt von der installierten Kapazität der KWK-Anlage ab und beträgt für Anlagen mit einer Kapazität kleiner 50 kW el 60.000 Vollbenutzungsstunden sowie die Hälfte für größere Anlagen. Für den angenommenen Planungszeitraum von 20 Jahren entspricht dies also einer jährlichen Vollbenutzungsstundenanzahl von 1.500 oder 3.000. Die entsprechende vergütete Elektrizitätsgestehungsmenge für Betriebsstunden der KWK-Anlage innerhalb dieser Anzahl wird in einer Nebenbedingung begrenzt, deren Rechte-Hand-Seite über Binärvariablen entsprechend des Leistungsbereichs der KWK-Anlage bestimmt wird.

\subsection{Technische Bewertungskennzahlen}

Zusätzlich zu den oben beschriebenen Modellergebnissen, werden weitere Indikatoren für die Bewertung von Energieautarkie sowie der Interaktion mit dem Elektrizitätsnetz ex-post berechnet. Nach McKenna et al. (2015) eignen sich zur Bewertung der Energieautarkie von Energiesystemen unter anderem zwei Indikatoren. Der Energieautarkiegrad setzt die eigenerzeugte und -verbrauchte Energie in das Verhältnis zur nachgefragten Energiemenge. In der vorliegenden Untersuchung erfolgt dabei eine Eingrenzung auf die Energieform der Elektrizität. Entsprechend wird der Energieautarkiegrad wie in Gleichung 6 definiert.

$$
E G_{e l}=\frac{\sum_{t=1}^{T} x_{e l, i}^{K W K}(t)}{\sum_{t=1}^{T} \operatorname{dem}_{e l}(t)}
$$

Der Eigenverbrauchsanteil repräsentiert überdies den Quotienten aus eigenerzeugter und -verbrauchter Elektrizität sowie der gesamten eigenerzeugten Elektrizitätsmenge. Die mathematische Formulierung dieser Bewertungskennzahl findet sich in Gleichung 7.

$$
E V_{e l}=\frac{\sum_{t=1}^{T} x_{e l, i}^{K W}(t)}{\sum_{t=1}^{T}\left(x_{e l, i}^{K W K}(t)+x_{e l, e}^{K W K}(t)\right)}
$$

Weiterhin stellt der Netzinteraktionsindex einen Indikator zur Quantifizierung der Wechselwirkungen zwischen von der KWK-Anlage in das Versorgungsnetz eingespeister und aus dem Netz bezogener Elektrizität dar (Salom 2011). Der Index wird durch die Standardabweichung der Netto-Elektrizitätsexporte bestimmt. Diese errechnen sich als Differenz der bezogenen und eingespeisten Elektrizitätsmenge in den Zeitschritten. Der normierte Netzinteraktionsindex ergibt sich schließlich durch die Division des Netzinteraktionsindex mit der Standardabweichung des Elektrizitätsnachfrageprofils. Der normierte Netzinteraktionsindex ist in Gleichung 8 formal beschrieben.

$$
N I I_{n o r m}=\frac{\sqrt{\frac{1}{T-1} \cdot \sum_{t=1}^{T}\left(\frac{E(t)}{\max (|E(t)|)}-\frac{1}{T} \cdot \sum_{t=1}^{T} \frac{E(t)}{\max (|E(t)|)}\right)^{2}}}{\sqrt{\frac{1}{T-1} \cdot \sum_{t=1}^{T}\left(\frac{\operatorname{dem}_{e l}(t)}{\max \left(\operatorname{dem}_{e l}(t)\right)}-\frac{1}{T} \cdot \sum_{t=1}^{T} \frac{\operatorname{dem}_{e l}(t)}{\max \left(\operatorname{dem}_{e l}(t)\right)}\right)^{2}}}
$$




\section{Modellannahmen und Anwendungsfälle}

In diesem Kapitel werden wesentliche Eingangsdaten für die Modellläufe aufgezeigt sowie Anwendungsfälle für die Instanzen des Modells beschrieben. Dazu wird zunächst auf die wesentlichen technischen, wirtschaftlichen sowie ökologischen Annahmen näher eingegangen, welche als Datenbasis für die Modellläufe dienen. Weiterhin werden als Anwendungsfälle Wohngebäude bzw. Wohngebäudestrukturen für die Modellrechnungen definiert.

\subsection{Datenbasis}

Für die Modellergebnisse zentrale Annahmen zu technischen, wirtschaftlichen und ökologischen Parametern werden in diesem Abschnitt dargelegt. In der Abbildung 2 sind die spezifischen Investitionen eines verbrennungsmotorischen BHKW, eines Gaskessels sowie eines Wärmespeichers hinterlegt. Dem Größendegressionseffekt folgend, nimmt die spezifische Investition mit zunehmender Anlagengröße ab. Im Optimierungsmodell werden diese Skaleneffekte mittels stückweiser linearer Approximation der degressiven Investitionsfunktion berücksichtigt.
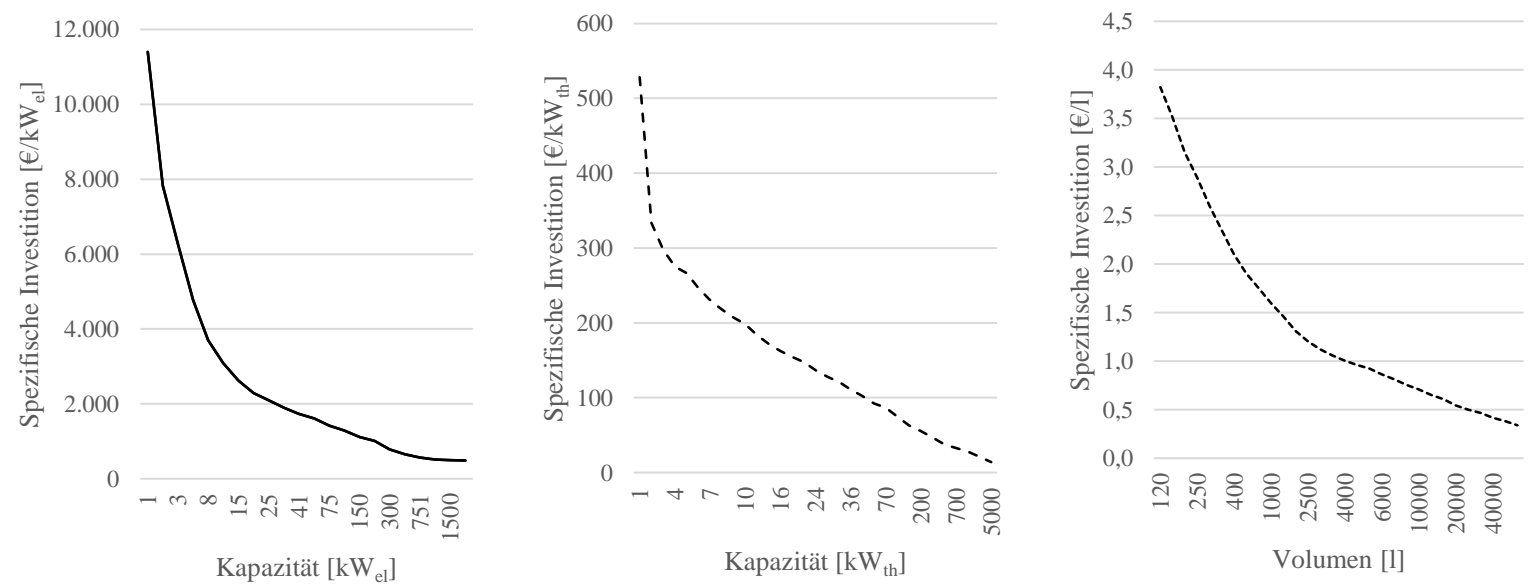

Abb. 2 Annahmen zur spezifischen Investition ausgewählter Technologien im Wärmesystem der Wohngebäude (v. 1. n. r. Verbrennungsmotor-BHKW, Gaskessel, Wärmespeicher) (Eigene Annahmen auf Basis von ASUE (2014), Bardt et al. (2014))

Weitere technische und wirtschaftliche Charakteristika der Technologien sowie Annahmen zu Primärenergie- und $\mathrm{CO}_{2}$-Emissionsfaktoren finden sich in Tabelle B.1 und Tabelle B.2.

Die Abbildung 3 skizziert die angenommene Entwicklung der Endverbraucherpreise von Elektrizität für Haushalte sowie den zeitlichen Verlauf der EEG-Umlage. Diese Annahmen sind der Studie zur Entwicklung der Energiemärkte von Schlesinger et al. (2014) entnommen. 


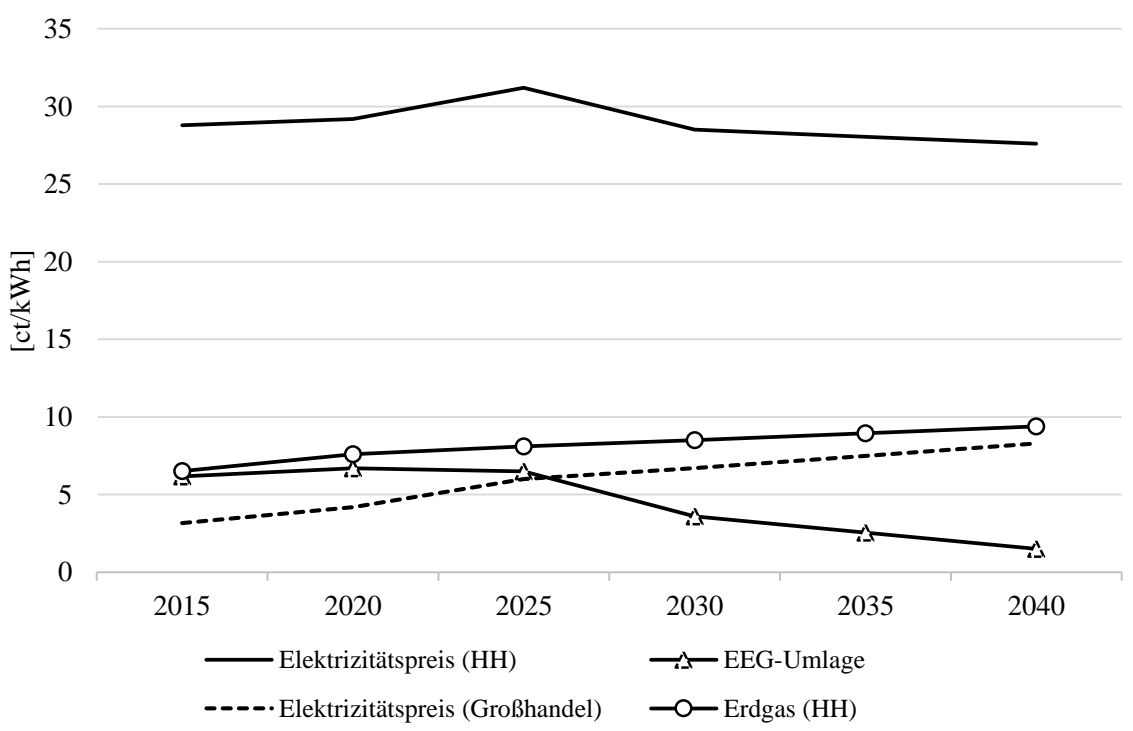

Abb. 3 Reale Entwicklung der Preise für Elektrizität und Erdgas sowie der EEG-Umlage (Schlesinger et al. (2014))

\subsection{Anwendungsfälle}

Für die Modellrechnungen werden verschiedene Nachfrageobjekte von Wohngebäuden definiert und diese hinsichtlich struktureller und energetischer Merkmale charakterisiert. Für die Modellanwendung besteht dabei ein Ziel darin, eine möglichst große Bandbreite des Wärme- und Elektrizitätsbedarfs abzubilden, um eine hinreichend große Fallzahl in den verschiedenen Leistungsklassen der KWKG-Neufassung zu erzielen und somit zu einem größeren Erkenntnisgewinn in der Bewertung der Neufassung zu gelangen. Im Sinne einer adäquaten Validierung des Optimierungsmodells existiert dabei ein Spannungsverhältnis zwischen einer hinreichend großen Anzahl der Fallstudien einerseits und einer handhabbaren Durchführung der Modelläufe vor dem Hintergrund einer erhöhten Modellkomplexität andererseits. Die Instanzen des Optimierungsmodells dezentraler Wärmeversorgungssysteme weisen je nach verwendetem Szenario bis zu ca. 250.000 Zeilen, ca. 900.000 Spalten und ca. 2.570.000 NonzeroElemente auf. Die Rechenzeiten für einen Modelllauf betragen in der GAMS/CPLEX-Entwicklungsumgebung bis zu ca. 11 Stunden. Zur Definition der Anwendungsfälle werden daher einzelne Ein- und Mehrfamilienhäuser definiert sowie diese auf verschiedenen räumlichen Ebenen zu einem Straßenzug, einem Block, einer Siedlung sowie einem Quartier aggregiert. ${ }^{2}$ Die Tabelle 2 gibt einen Überblick über die definierten Nachfrageobjekte in den Anwendungsfällen der Modellrechnungen.

Tab. 2 Überblick über die Anwendungsfälle der Modellrechnungen

\begin{tabular}{|l|l|l|l|l|}
\hline $\begin{array}{l}\text { Anwendungsfall/ } \\
\text { Nachfrageobjekt }\end{array}$ & Fallbezeichnung & $\begin{array}{l}\text { Anzahl } \\
\text { Haushalte }\end{array}$ & $\begin{array}{l}\text { Jahresheizwärme- } \\
\text { bedarf }\left(\mathrm{MWh}_{\mathrm{th}} / \mathrm{a}\right)\end{array}$ & $\begin{array}{l}\text { Jahreselektrizitäts- } \\
\text { bedarf }(\mathrm{MWh} / \mathrm{a})\end{array}$ \\
\hline Einfamilienhaus & EFH_1a & 1 & 17,5 & 2,29 \\
\hline
\end{tabular}

\footnotetext{
${ }^{2}$ Dabei ist zu beachten, dass die notwendigen Ausgaben für den Wärmenetzausbau bzw. die -erweiterung bei den größeren Anwendungsfällen nicht berücksichtigt werden. Da in dem aktuellen Beitrag jedoch der Vergleich der drei unterschiedlichen Szenarien (Referenz, KWKG 2012, KWKG 2016) im Fokus steht, wird dieser Sachverhalt hinsichtlich des Einflusses auf die Ergebnisse als weniger relevant erachtet. Zudem wird von ggfs. notwendigen Maßnahmen am elektrischen (Verteil-)Netz, wie dessen Ausbau oder die Installation technischer Betriebsmittel im Modellierungsansatz ebenso abstrahiert. Letztlich muss auch berücksichtigt werden, dass insbesondere die Versorgung der Siedlung und des Quartiers mit einer zentralen KWK-Anlage eine vereinfachte Annahme eines realistischen Betreibermodells darstellt.
} 


\begin{tabular}{|l|l|l|l|l|}
\hline Einfamilienhaus $^{3}$ & EFH_1b & 1 & 17,5 & 2,92 \\
\hline Straßenzug & EFH_13 & 13 & 227,6 & 29,8 \\
\hline Mehrfamilienhaus & MFH_13 & 13 & 55,4 & 29,8 \\
\hline Block & EFH_100 & 100 & $1.750,8$ & 229,0 \\
\hline Block & MFH_100 & 100 & 426,5 & 229,0 \\
\hline Siedlung & EFH_204 & 204 & $3.573,2$ & 467,1 \\
\hline Quartier & EFH_1000 & 1000 & $17.508,4$ & $2.289,7$ \\
\hline
\end{tabular}

Die Nachfrage nach Elektrizität errechnet sich in Höhe und zeitlichem Verlauf entsprechend den Berechnungen des stochastischen Simulationsmodells in McKenna et al. (2016). Die Nachfrage nach Raumwärme und Warmwasser stützt sich hingegen auf Ergebnisse eines Modells zur thermischen Gebäudesimulation. Ferner erfolgt in dem Modell eine zeitliche Disaggregation der jährlichen Bedarfe mittels eines Lastprofils. Dabei kommt es zu einer Unterteilung des Jahres in insgesamt 6.048 Zeitschritte, welche sich auf eine Auswahl von neun Typwochen aus den drei Jahreszeiten Sommer, Winter und Übergangsjahreszeit in 15-minütiger Auflösung ergeben. Die Marktdaten für die stündlichen Spotmarktpreise für Elektrizität wie auch die vierteljährlichen durchschnittlichen Preise für Grundlaststrom an der EEX entstammen dem Verlauf aus dem Jahr 2015.

\section{Modellergebnisse und Diskussion}

In diesem Kapitel werden Ergebnisse der Modellierung zur Bewertung der KWKG-Neufassung 2016 dargelegt. Dazu werden die wesentlichen Ergebnisgrößen, welche aus den Modellläufen gewonnen werden, aufgezeigt und diskutiert sowie eine Auswahl dieser Größen einer Sensitivitätsanalyse unterzogen. Das Kapitel schließt mit einer kritischen Würdigung des entwickelten Ansatzes. Es ist dabei zu beachten, dass sich alle Ergebnisse auf das Jahr 2016 beziehen, also von einer Inbetriebnahme der KWK-Systeme in diesem Jahr mit den im Jahr 2016 vorherrschenden Rahmenbedingungen ausgehen ${ }^{4}$.

\subsection{Ergebnisse der Modellrechnungen}

Die Ergebnisse der Modellrechnungen werden in den folgenden Unterabschnitten weiter nach deren wirtschaftlichen, technischen sowie ökologischen Implikationen inhaltlich strukturiert. Zudem findet sich ein Abschnitt mit Ergebnissen zur Einsatzplanung eines KWK-Systems.

\subsubsection{Ergebnisse zur Wirtschaftlichkeit}

Die Zielfunktion beschreibt die Annuität der Jahresgesamtausgaben der Versorgung des Nachfrageobjekts mit Elektrizität und Wärme. Entsprechend können diese Ausgaben als Ergebnis der Modellberechnungen ausgewiesen werden. Die Abbildung 4 stellt hierzu die Jahresgesamtausgaben pro Haushalt für die definierten Anwendungsfälle

\footnotetext{
${ }^{3}$ Das Einfamilienhaus EFH_1b unterscheidet sich von dem Einfamilienhaus EFH_1a in der Bewohnerstruktur. So weist das EFH_1a bzw. EFH_1b eine Bewohneranzahl von 2 bzw. 4 auf.

${ }^{4}$ Nichtsdestoweniger wird die zeitdynamische Entwicklung der Preise und EEG-Umlage in den Berechnungen mit dem Basisjahr 2016 einbezogen (vgl. Kapitel 3.1).
} 
unter den Fördermechanismen des KWKG 2012 und des KWKG 2016 sowie für den Referenzfall (ungekoppelte Elektrizitäts- und Wärmeversorgung) für das Bezugsjahr 2016 dar.

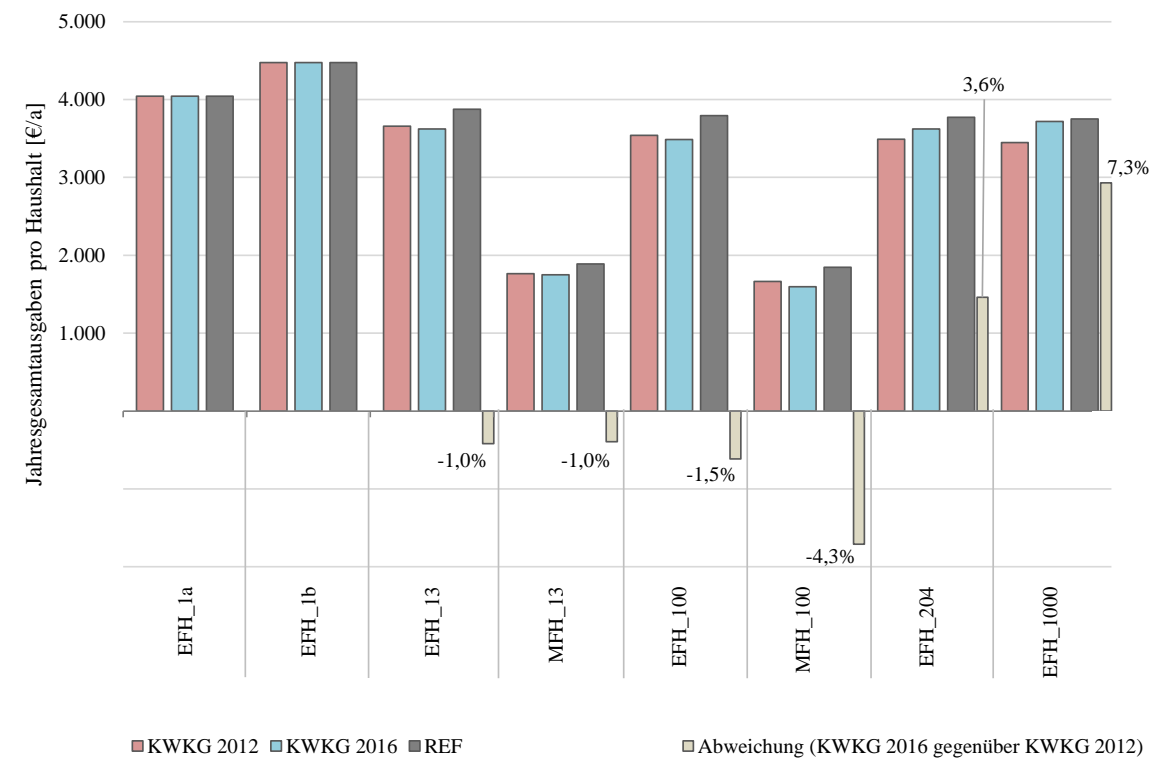

Abb. 4 Jahresgesamtausgaben pro Haushalt der Anwendungsfälle nach dem KWKG 2012 und dem KWKG 2016 sowie für den Referenzfall für das Bezugsjahr 2016

Aus den Modellergebnissen können mehrere Schlussfolgerungen abgeleitet werden. Zum einen erweist sich das Konzept der gekoppelten Erzeugung von Elektrizität und Wärme gegenüber der ungekoppelten Versorgung für alle Anwendungsfälle bis auf die Nachfrageobjekte einzelner Einfamilienhäuser („EFH_1a“ und „EFH_1b“) als ökonomisch vorteilhaft. Für Letztere entspricht das Ergebnis für die optimale Elektrizitäts- und Wärmeversorgung für beide Fassungen des KWKG dem Ergebnis des Referenzfalls. Dies liegt daran, dass in der Modellversion der KWKG-Regime nicht notwendigerweise eine KWK-Anlage gewählt werden muss, sondern deren Kapazität auch zu Null gesetzt werden kann. Die Nichtberücksichtigung der Kraft-Wärme-Kopplung in diesen Fällen ist u. a. auf den zu geringen Verbrauch von Elektrizität und vor allem dem zu geringen Wärmebedarf zurückzuführen. Wie anhand des Nachfrageobjekts „EFH_1b“ ersichtlich wird, führt auch eine erhöhte elektrische Nachfrage nicht zu einer wirtschaftlichen Verbesserung durch den Einsatz einer KWK-Anlage. Zum anderen ist eine Degression der spezifischen Jahresgesamtausgaben mit zunehmender Energienachfrage der betrachteten Anwendungsfälle zu erkennen. Dies ist auf Größendegressionseffekte bei der Anlageninvestition zurückzuführen. Eine Ausnahme stellen hierbei die Mehrfamilienhäuser sowie die beiden Nachfrageobjekte Siedlung und Quartier unter den Regelungen des KWKG 2016 dar. Bei den MFH-Objekten ist der beträchtlich geringere Jahresheizwärmebedarf gegenüber den EFH-Objekten mit gleicher Haushaltsanzahl als Grund für die Degression anzuführen. Dagegen nehmen die spezifischen Ausgaben für die Siedlung und das Quartier unter den Regelungen der neuen KWKGFassung zu. Letzteres Ergebnis ist darauf zurückzuführen, dass im Vergleich zu dem Straßenzug und dem Block keine weiteren Größendegressionseffekte hinsichtlich der Investition erzielt werden. Dieser Sachverhalt wird nachfolgend in der Analyse der installierten Kapazität eingehender untersucht.

Zudem kann die Abweichung der spezifischen Jahresgesamtausgaben und damit die ökonomische Vorteilhaftigkeit unter den beiden Fassungen des KWKG näher untersucht werden. Die Abbildung 4 stellt hierzu auch die prozentuale Abweichung der Jahresgesamtausgaben nach dem KWKG 2016 bezogen auf diejenigen nach dem KWKG 2012 dar. Für die untersuchten Wohngebäudestrukturen der mittleren Aggregationsebenen werden 
demnach jährliche Einsparungen von ca. 1,0 \% bis ca. 4,3\% erzielt. Hingegen kommt es zu einer Umkehr der Vorteilhaftigkeit für größere zentral aus KWK versorgte Strukturen (Siedlung und Quartier). Der Vergleich der Zielfunktionswerte zeigt um ca. 3,6 \% bzw. ca. 7,3\% verminderte Jahresgesamtausgaben unter den Regelungen des KWKG 2012 gegenüber den neuen Regelungen des KWKG 2016.

Des Weiteren können die Einzahlungen im Zusammenhang mit dem KWK-Einsatz hinsichtlich ihrer Zusammensetzung bzw. Mittelherkunft weiter untersucht werden. Hierzu weist die Abbildung 5 für die untersuchten Fälle mit KWK-Installation die Zahlungsart differenziert nach Zuschlag sowie Vergütung aus. Die Zahlungen aus dem Zuschlag werden ferner dahingehend unterschieden, ob sie bei der Selbstnutzung oder Netzeinspeisung der erzeugten Elektrizität aus KWK anfallen. Die Vergütung umfasst die Zahlungen der abnehmenden Netzbetreiber von netzeingespeister Elektrizität für die kaufmännische Abnahme bzw. Direktvermarktung zu EPEX-Spotpreisen.

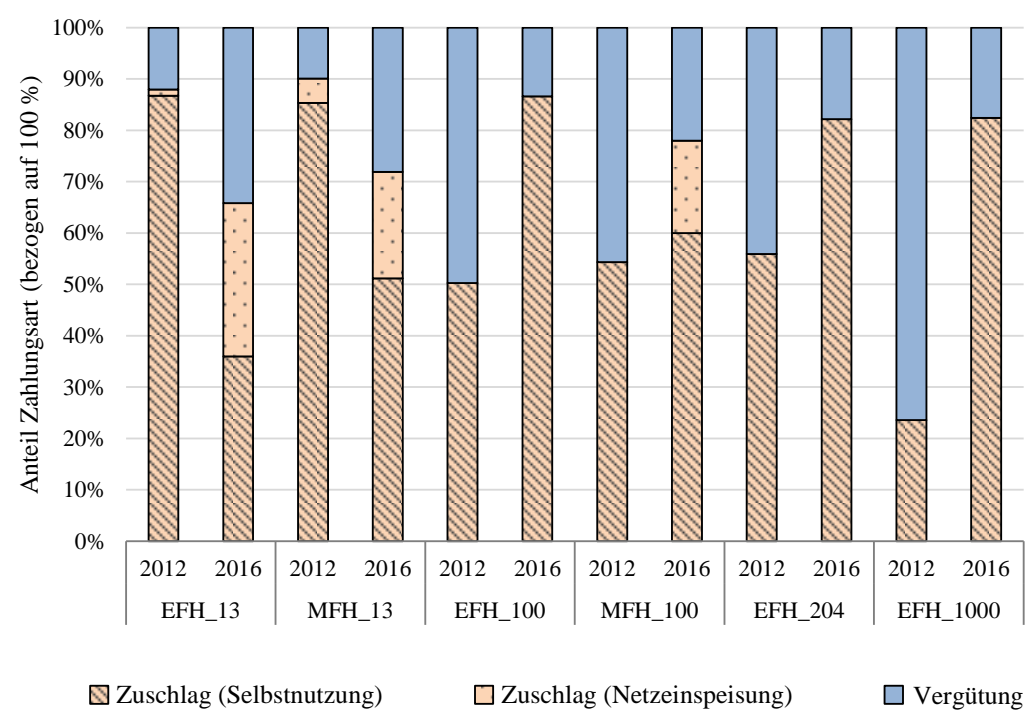

Abb. 5 Zusammensetzung der Einzahlungen (Zuschlag, Vergütung) für die Anwendungsfälle unter dem KWKG 2012 und 2016 für das Bezugsjahr 2016

Aus der Abbildung können mehrere Schlussfolgerungen gezogen werden. Zum einen tragen die gesetzlichen Zuschlagzahlungen finanziert durch KWK-Umlage in der Mehrzahl der Anwendungsfälle unter den Regelungen des KWKG 2016 den größten Anteil an der gesamten Einzahlung bei. Mit Ausnahme des Falles „EFH_1000“ unter den Rahmenbedingungen des KWKG 2012 beträgt dieser Anteil mindestens $50 \%$ und erreicht einen Spitzenwert von ca. 90,1\% (Fall „MFH_13“ unter KWKG 2012). Der Einfluss der Erhöhung der Förderbeträge für die Netzeinspeisung im KWKG 2016 wird dabei in den beiden Fällen mit den 13 Haushalten besonders deutlich, in denen der Anteil der Zuschläge am höchsten ist. Während sich der Anteil der Zuschläge für die Ergebnisse unter den Regelungen des KWKG 2016 auf Grund des fallübergreifenden Verbleibs in derselben Leistungsklasse in einem geringeren Intervall zwischen ca. 65,8\% und ca. 86,8 \% bewegt, fällt die Bandbreite unter dem Regime des KWKG 2012 hingegen deutlich größer aus. Mit dem Erreichen einer höheren Leistungsklasse mit geringerem spezifischem Zuschlag ab dem Fall „EFH_100“ wird unter dem KWKG 2012 eine 
deutliche Verschiebung hin zur marktbasierten Vergütung vollzogen. Die für die Anwendungsfälle in den Modellläufen bestimmten Erzeugungskapazitäten werden im nachfolgenden Abschnitt weiter untersucht.

\subsubsection{Ergebnisse zu technischen Aspekten}

Eine zentrale Eigenschaft des Modells ist die Auslegung der Systemkomponenten Verbrennungsmotor-BHKW und Spitzenlastkessel. In der Abbildung 6 wird die durch die Optimierung determinierte elektrische Kapazität der KWK-Anlagen veranschaulicht. Weiterhin sind auch die implizierten jährlichen Vollbenutzungsstunden abgebildet. Aus Gründen der Übersichtlichkeit sind diese Kenngrößen für den Anwendungsfall „EFH_1000“ in einem separaten Teilbild dargestellt.

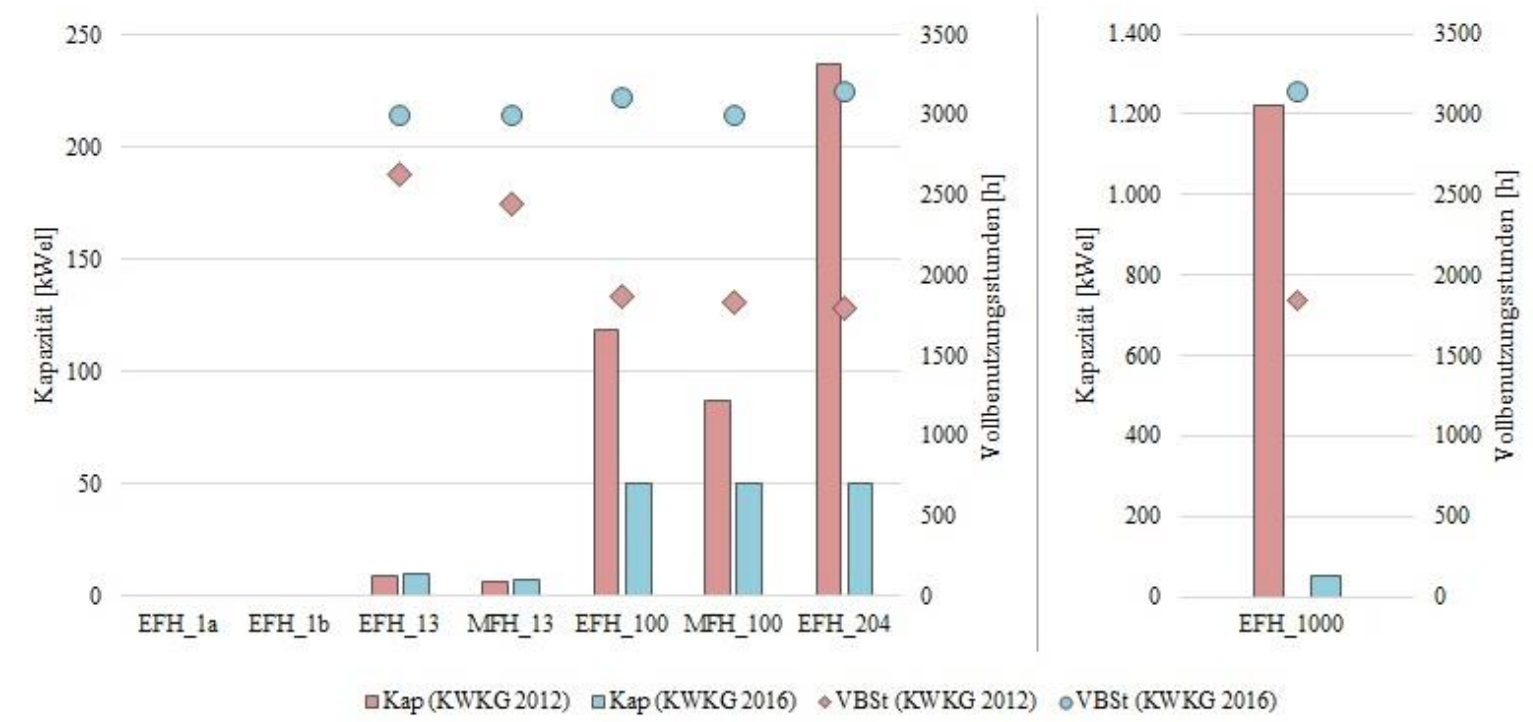

Abb. 6 Installierte elektrische Kapazitäten der KWK-Anlagen sowie jährliche Vollbenutzungsstunden der Anwendungsfälle unter den Regelungen des KWKG 2012 und des KWKG 2016 für das Bezugsjahr 2016

Aus der Dimensionierung der KWK-Anlage lassen sich für die Anwendungsfälle einige zentrale Erkenntnisse ableiten. So fällt die Anlagenkapazität für die Fälle „EFH_13“ und „MFH_13“ unter dem KWKG 2016 geringfügig größer aus als unter dem KWKG $2012\left(10,0 \mathrm{~kW}_{\text {el }}\right.$ gegenüber 8,61 kW el bzW. 7,19 kW $\mathrm{kl}_{\text {el }}$ gegenüber $6,65 \mathrm{~kW}_{\mathrm{el}}$ ). Dieses Verhältnis kehrt sich für die höheren Aggregationsebenen hingegen um. Demzufolge bleibt unter dem Vergütungsregime des KWKG 2016 die Anlagenkapazität der KWK-Einheit konstant bei 50 kW el für alle weiteren Anwendungsfälle. Die Grenze der 60.000 Vollbenutzungsstunden bei $50 \mathrm{~kW}_{\mathrm{el}}$ erweist sich für die gewählten Fallbeispiele also als von entscheidender Bedeutung. Entsprechend liegen die jährlichen Vollbenutzungsstunden unter dem KWKG 2016-Regime bei exakt 3.000 bzw. für die Objekte größeren Verbrauchs etwas darüber.

Die Modellergebnisse unter dem KWKG 2012 zeigen für den untersuchten hohen Verbrauchsbereich des Blocks, der Siedlung und des Quartiers hingegen eine höhere Dimensionierung der Anlagen bei gleichzeitig gegenüber dem KWKG 2016-Regime niedrigeren Vollbenutzungsstunden auf (vgl. Abb. 6). Insgesamt zeigt sich also eine gegenläufige Abhängigkeit der Vollbenutzungsstundenzahl und Erzeugungsleistung.

Zudem können aus der Analyse Schlüsse zu dem Einfluss der Befreiung von der EEG-Umlage gezogen werden. Unter dem KWKG 2012 überschreitet die implizierte jährliche Anzahl der Vollbenutzungsstunden für die Fälle 
„EFH_100“ bis „EFH_204“ die Anzahl der zuschlagberechtigten jährlichen Betriebsstunden von $1.500^{5}$ in erkennbarem Maße. Für den mittleren Verbrauchsbereich („EFH_13“ und „MFH_13“) liegt diese dagegen merklich erhöht bei ca. 2.625 und 2.440. Die Erhöhung der Anzahl um ca. 1.000 Stunden ist der Befreiung von der Zahlung der EEG-Umlage für selbsterzeugte und -verbrauchte Elektrizität geschuldet, welche annahmegemäß für Anlagen mit einer Kapazität kleiner $10 \mathrm{~kW}_{\text {el }}$ für eine Erzeugungsmenge der Elektrizität in Höhe von $10 \mathrm{MWh}$ pro Jahr greift. Die Befreiung von der EEG-Umlage impliziert unter dem KWKG 2012 also aus Gründen einer erhöhten Wirtschaftlichkeit eine Mehrproduktion von Elektrizität und Wärme. Unter dem KWKG 2016 wird die implizierte Vollbenutzungsstundenanzahl von 3.000 für den mittleren Verbrauchsbereich dagegen nicht überschritten. Ein Grund hierfür könnte sein, dass eine Mehrerzeugung der Wärme die thermische Nachfrage entsprechend übererfüllen und somit insgesamt zu einem wirtschaftlichen Nachteil führen würde.

Für die Mehrfamilienhäuser wird hingegen ersichtlich, dass die KWK-Anlagen im Vergleich zu den Einfamilienhäusern mit gleicher Anzahl an Haushalten unter den beiden Vergütungsregimes geringer dimensioniert werden, was auf den vergleichsweise geringen Wärmebedarf zurückzuführen ist. Eine Ausnahme bildet hierbei der Häuserblock unter dem KWKG 2016-Regime, für den die Anlagenkapazität in beiden Fällen der 100 Haushalte ca. $50 \mathrm{~kW}_{\mathrm{el}}$ beträgt.

In dem Modell wird ebenso die Vermarktungsoption für netzeingespeiste Elektrizität nach KWKG 2016 in der Optimierung berücksichtigt (vgl. Kapitel 2.4). Es zeigt sich aus den Modellläufen, dass in fast allen Anwendungsfällen die Direktvermarktungsoption präferiert wird. Eine Ausnahme bildet der Fall „MFH_13““, in welchem die kaufmännische Abnahme auf Basis des vierteljährlich konstanten Durchschnittspreises für Grundlaststrom an der EEX gewählt wird.

Neben der KWK-Anlage wird auch der Spitzenlastkessel ebenso wie der Wärmespeicher in den Modelläufen für die Nachfrageobjekte dimensioniert. Die Abbildung 7 weist die korrespondierenden thermischen Kapazitäten bzw. Volumina aus.

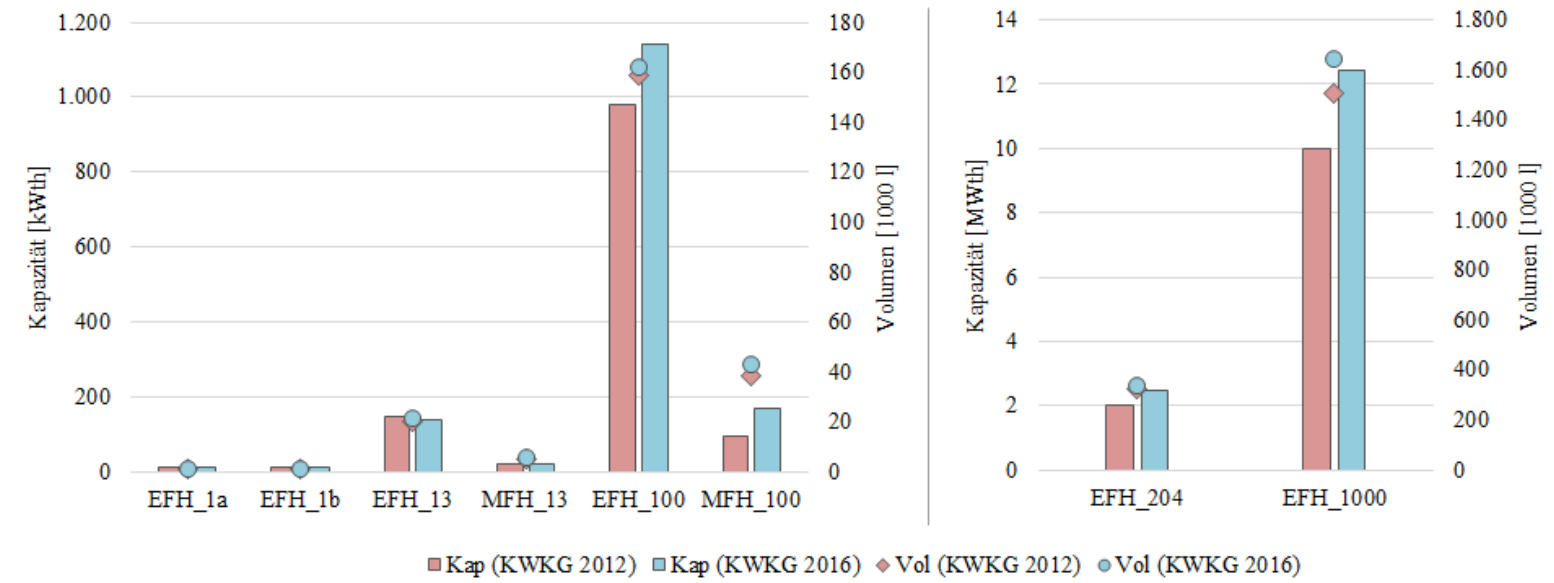

Abb. 7 Installierte thermische Kapazitäten der Gaskessel sowie Volumen der Wärmespeicher der Fälle nach KWKG 2012 und 2016 für das Bezugsjahr 2016

\footnotetext{
${ }^{5}$ Bei der Annahme einer Nutzungsdauer von 20 Jahren und gleichmäßiger Verteilung auf die Betriebsjahre.
} 
Die Kapazität der Gaskessel fällt unter den Regelungen des KWKG 2016 für den Straßenzug geringer und für den Häuserblock, die Siedlung sowie das Quartier höher aus. Die Abbildungen 6 und 7 zeigen, dass sich die thermischen Kapazitäten der KWK-Anlage und des Spitzenlastkessel zur Deckung der Nachfrage nach der gesamten Wärmenachfrage in komplementärer Weise ergänzen. Zudem wird in allen Anwendungsfällen der thermische Speicher unter dem KWKG 2016-Regime größer dimensioniert als unter den Bedingungen des KWKG 2012. Dies spiegelt einerseits den erhöhten Bedarf nach einer Verschiebung der auf Grund der geringeren Leistung gleichmäßigeren thermischen Erzeugung in Zeitschritte des hohen thermischen Verbrauchs wider. Andererseits ermöglicht es auch eine marktorientiertere Fahrweise durch Ausnutzen von Preisspitzen bei auf Grund mangelnden zeitgleichen Wärmebedarfs notwendiger Speicherung der erzeugten Wärme.

Ebenso können weitere technische Ergebnisgrößen des Optimierungsmodells untersucht werden. Der normierte Netzinteraktionsindex beschreibt das Maß des Elektrizitätsaustausches mit dem Versorgungsnetz und ist in der Abbildung 8 für die Anwendungsfälle dargestellt.

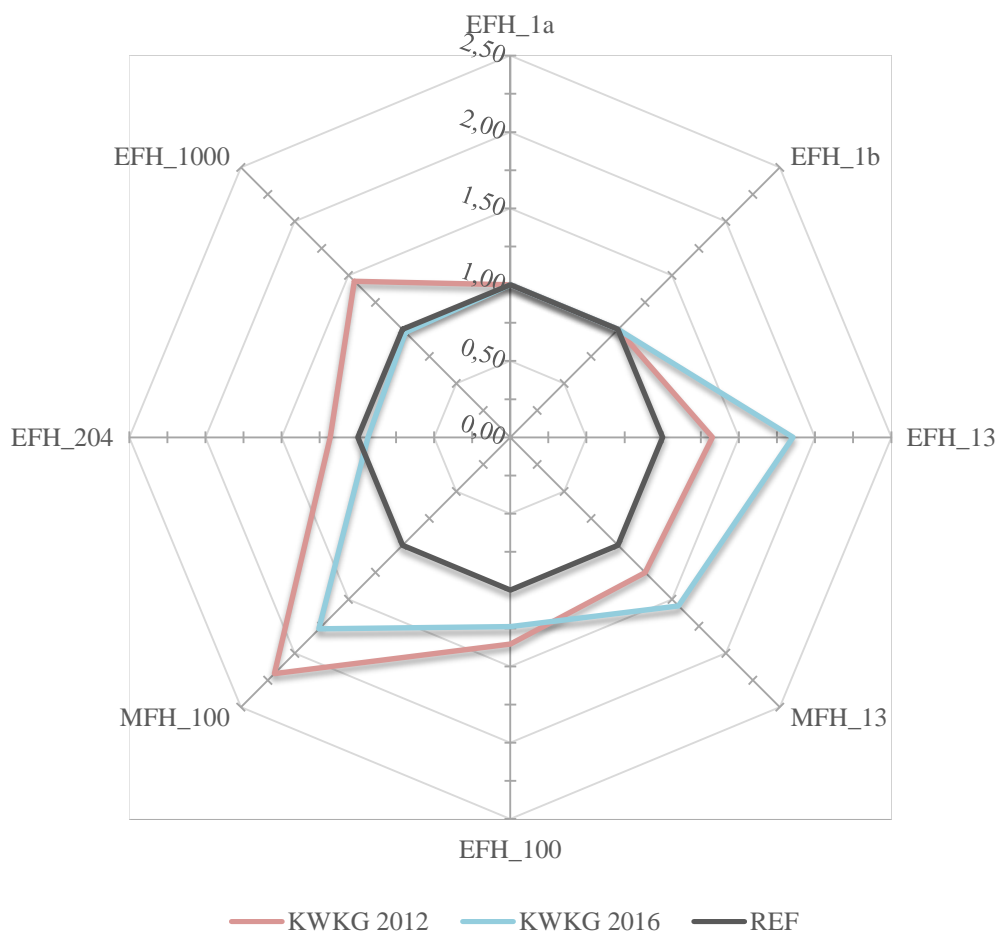

Abb. 8 Netzinteraktion der Fälle nach KWKG 2012 und 2016 sowie des Referenzfalls für das Bezugsjahr 2016

Für den Referenzfall stimmt das Elektrizitätsverbrauchsprofil mit dem Elektrizitätsbezugsprofil aus dem Netz überein, da definitionsgemäß die einzige Möglichkeit zur Nachfragedeckung der Elektrizitätsbezug aus dem Netz darstellt, da keine KWK-Anlagen installiert werden können. Dementsprechend nimmt der Netzinteraktionsindex für alle Anwendungsfälle im Referenzfall den Wert 1 an (vgl. Abb. 8). Hingegen kann für das Vergütungsregime nach KWKG 2012 bzw. KWKG 2016 keine gleichgerichtete Aussage hinsichtlich der Netzinteraktion getroffen werden. Für den mittleren Verbrauchsbereich des Straßenzugs ist der Index unter dem KWKG 2016 gegenüber dem KWKG 2012 erhöht (1,85 gegenüber 1,33 bei „EFH_13“ und 1,56 gegenüber 1,25 bei „MFH_13“). Dies liegt 
in der höheren Auslegung der KWK-Anlagen unter der Neufassung des KWKG begründet. Bei ansonsten gleichem Elektrizitätsverbrauch führt dies also zu einer erhöhten Erzeugung und mangels Eigennutzungsmöglichkeit zu einer gesteigerten Einspeisung in das Versorgungsnetz. Die höhere Auslegung ist in diesem Fall also durch den vergleichsweise hohen Zuschlag i. H. v. 8,00 ct/kWh für netzeingespeiste Elektrizität motiviert. Für den hohen Verbrauchsbereich des Häuserblocks, der Siedlung und des Quartiers bleibt der Netzinteraktionsindex unter dem KWKG 2016 jedoch hinter demjenigen unter dem KWKG 2012 zurück. Dies ist dagegen mit der durchweg vergleichsweise geringeren Kapazität der KWK-Anlagen zu begründen, bei der die erzeugte Elektrizität zumeist selbstgenutzt werden kann und somit kein Austausch mit dem Netz stattfindet.

Die Modellergebnisse können ebenso vor dem Hintergrund des Energieautarkiegrades sowie dem Eigenverbrauchsanteil der Elektrizität diskutiert werden. Hierzu weist die Abbildung 9 den Autarkiegrad sowie den Eigenverbrauchsanteil der betrachteten Anwendungsfälle in den Modellderivaten des KWKG 2012 und des KWKG 2016 bezogen auf den Energieträger Elektrizität aus.

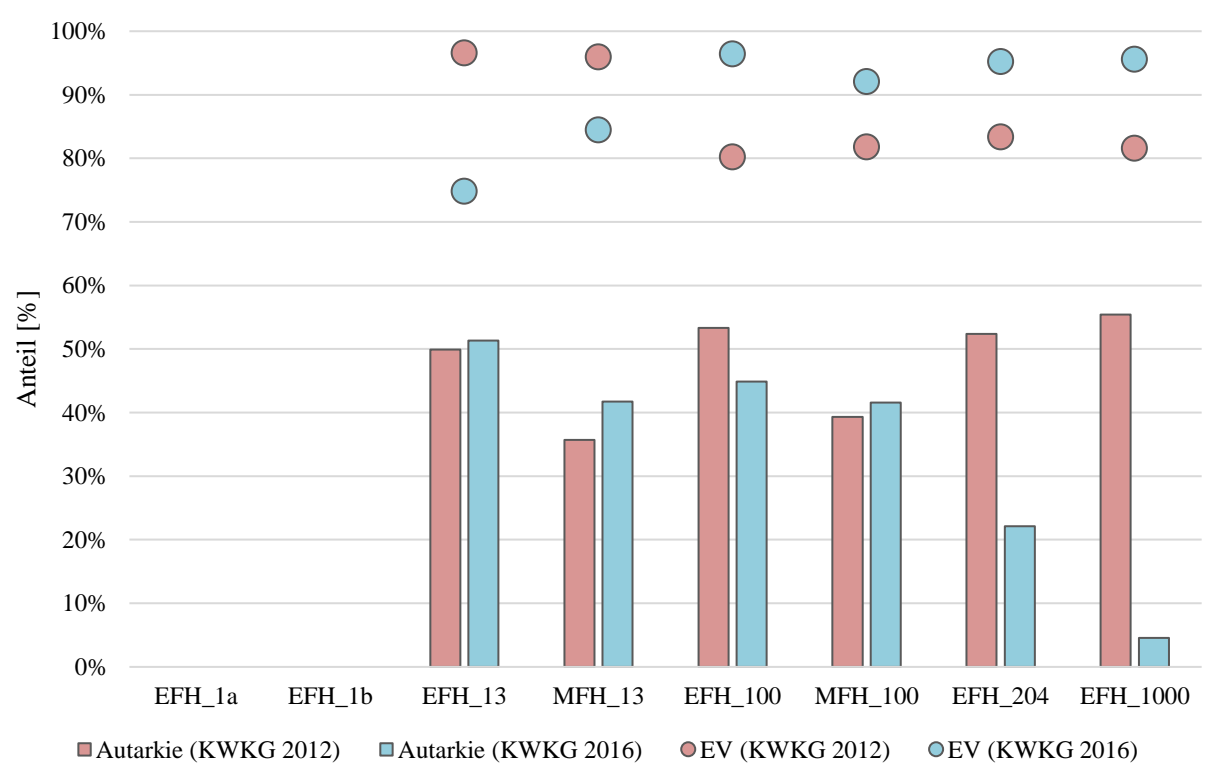

Abb. 9 Autarkiegrad sowie Eigenverbrauchsanteil der Fälle nach KWKG 2012 und 2016 für das Bezugsjahr 2016

Auch aus diesen technischen Bewertungskennzahlen des optimierten Wärmeversorgungssystems können wichtige Aussagen abgeleitet werden. In den beiden Gebäudeobjekten mit 13 Haushalten ist der Autarkiegrad unter dem KWKG 2016 gegenüber der Vorgängerversion des Gesetzes erhöht (ca. $51 \%$ gegenüber $50 \%$ bzw. ca. $42 \%$ gegenüber $36 \%$ ). Gleichwohl ist der Eigenverbrauchsanteil verringert (ca. $75 \%$ gegenüber $97 \%$ bzw. ca. $84 \%$ gegenüber $96 \%$ ). Dies deckt sich also mit den Erkenntnissen bezüglich der Netzinteraktion den Anteil der eingespeisten Elektrizität betreffend. Mit Ausnahme des Falles „MFH_100“ ist der Autarkiegrad unter dem KWKG 2016 hingegen gegenüber dem KWKG 2012 verringert, wobei die Abweichung mit steigender Aggregationsebene zunimmt. Als Grund hierfür lässt sich der bei gleichbleibender KWK-Anlagenkapazität zunehmende Bedarf an Elektrizität aus dem Versorgungsnetz anführen. Zugleich erhöht sich unter den Regelungen des KWKG 2016 der Eigenverbrauchsanteil mit Größe des Verbrauchsobjekts, da bei steigendem 
Elektrizitätsverbrauch und konstanter Anlagengröße das Verbrennungsmotor-BHKW zunehmend der Charakter einer Grundlast-Technologie zukommt.

\subsubsection{Ergebnisse zu ökologischen Aspekten}

Des Weiteren lassen sich auch Ergebnisse im Hinblick auf ökologische Aspekte aus den Modellrechnungen ableiten. Hierzu weist die Abbildung 10 die $\mathrm{CO}_{2}$-Emissionen im Bezugsjahr 2016 auf Basis der direkten und indirekten Emissionsfaktoren für sämtliche Anwendungsfälle auf Grundlage des spezifischen Emissionsniveaus der Energieträger im Jahr 2016 aus. Weiterhin werden die Förderregelungen beider KWKG-Fassungen sowie der Referenzfall differenziert. Zudem wird der Ausstoß des untersuchten Treibhausgases, welcher unmittelbar auf die Energiebedarfsdeckung der Nachfrageobjekte entfällt, von der im Energiesystem außerhalb des betrachteten Systems vermiedenen $\mathrm{CO}_{2}$-Emission unterschieden. Letztere quantifiziert die Verdrängung bzw. Substitution von Elektrizität des nationalen Erzeugungsmixes durch Elektrizität der KWK-Anlage in Bezug auf den $\mathrm{CO}_{2}-$ Ausstoß.

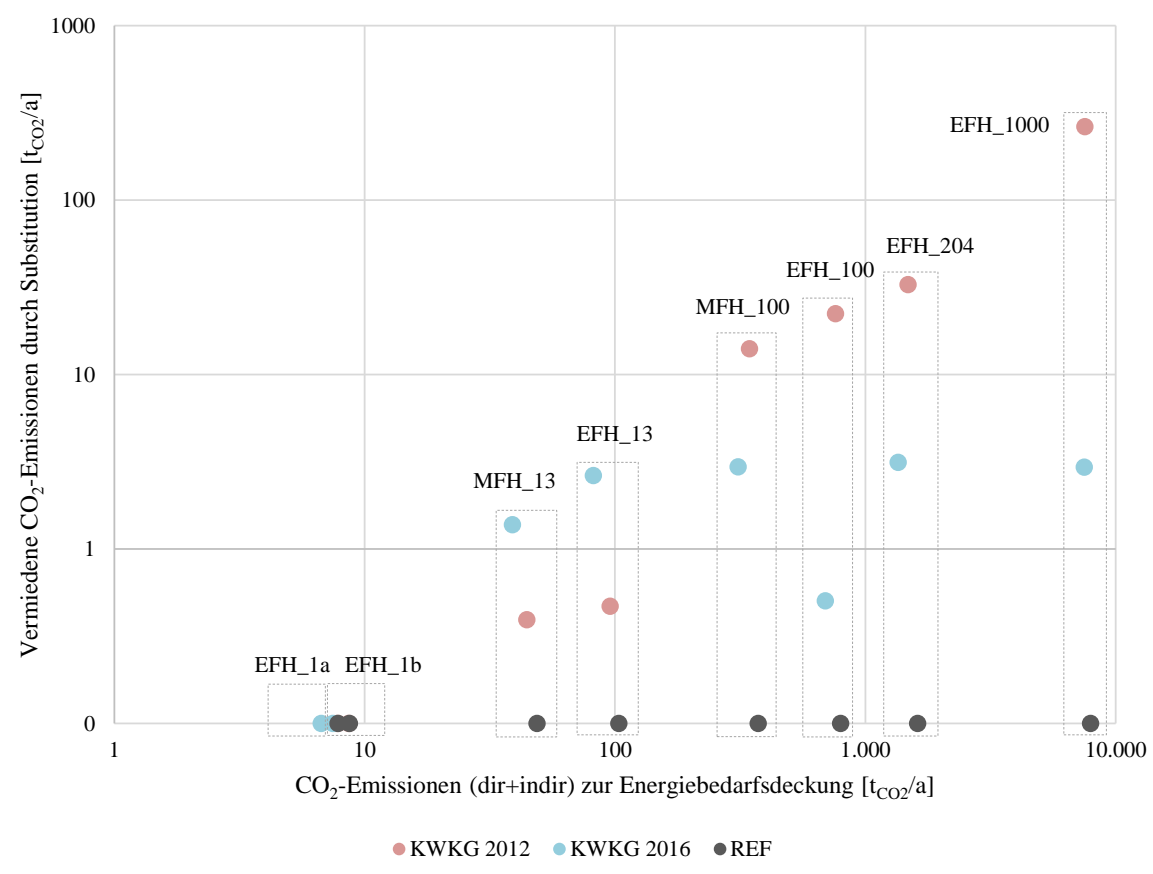

Abb. $10 \mathrm{CO}_{2}$-Emissionen der Fälle nach KWKG 2012 und 2016 sowie im Referenzfall für das Bezugsjahr 2016

Auch hinsichtlich der $\mathrm{CO}_{2}$-Emission (im Bezugsjahr 2016) ergibt sich eine Reihe von Schlussfolgerungen aus den Optimierungsläufen. So weisen in allen Anwendungsfällen die gekoppelte Versorgungsvariante geringere $\mathrm{CO}_{2}-$ Emissionen als die ungekoppelte Versorgungsvariante auf. Hinsichtlich der vermiedenen Emission werden wieder die zuvor untersuchten Verhältnisse der eingespeisten und der selbstgenutzten Elektrizität deutlich. Demzufolge verdrängen für den Straßenzug die KWK-Anlagen unter dem KWKG 2016 auf Grund der im Vergleich höheren Einspeisung die von dem konventionellen Kraftwerkspark erzeugte Elektrizität in größerem Maße als unter dem KWKG 2012 (ca. 2,53 t $\mathrm{t}_{\mathrm{CO} 2} /$ a gegenüber ca. 0,37 t $\mathrm{t}_{\mathrm{CO} 2} / \mathrm{a}$ bei „EFH_13“ und ca. 1,28 $\mathrm{t}_{\mathrm{CO} 2 / \mathrm{a}}$ gegenüber ca. 0,29 $\mathrm{t}_{\mathrm{CO} 2} / \mathrm{a}$ bei „MFH_13“). Dieses Verhältnis wird hingegen für die höheren Aggregationsebenen umgekehrt, so dass für den 
Fall des Quartiers unter dem KWKG 2012 eine Verdrängung von ca. 263,1 t $\mathrm{t}_{\mathrm{CO} 2} / \mathrm{a}$ erreicht wird (2,85 $\mathrm{t}_{\mathrm{CO} 2} / \mathrm{a}$ unter dem KWKG 2016).

\subsubsection{Ergebnisse zur Einsatzplanung}

In dem vorgestellten Optimierungsmodell dezentraler Wärmeversorgungssysteme wird neben der ausgabenminimalen Dimensionierung der betrachteten Technologien ebenso deren optimaler Einsatz auf der zeitlich hochaufgelösten Ebene bestimmt. In der Abbildung 11 werden der Zeitverlauf der thermischen und elektrischen Nachfrage, die Energieausbringung der KWK-Anlage sowie die EPEX-Spotpreise für den Anwendungsfall des Straßenzugs der Einfamilienhäuser in den Werktagen einer Typwoche im Winter beispielhaft dargestellt.

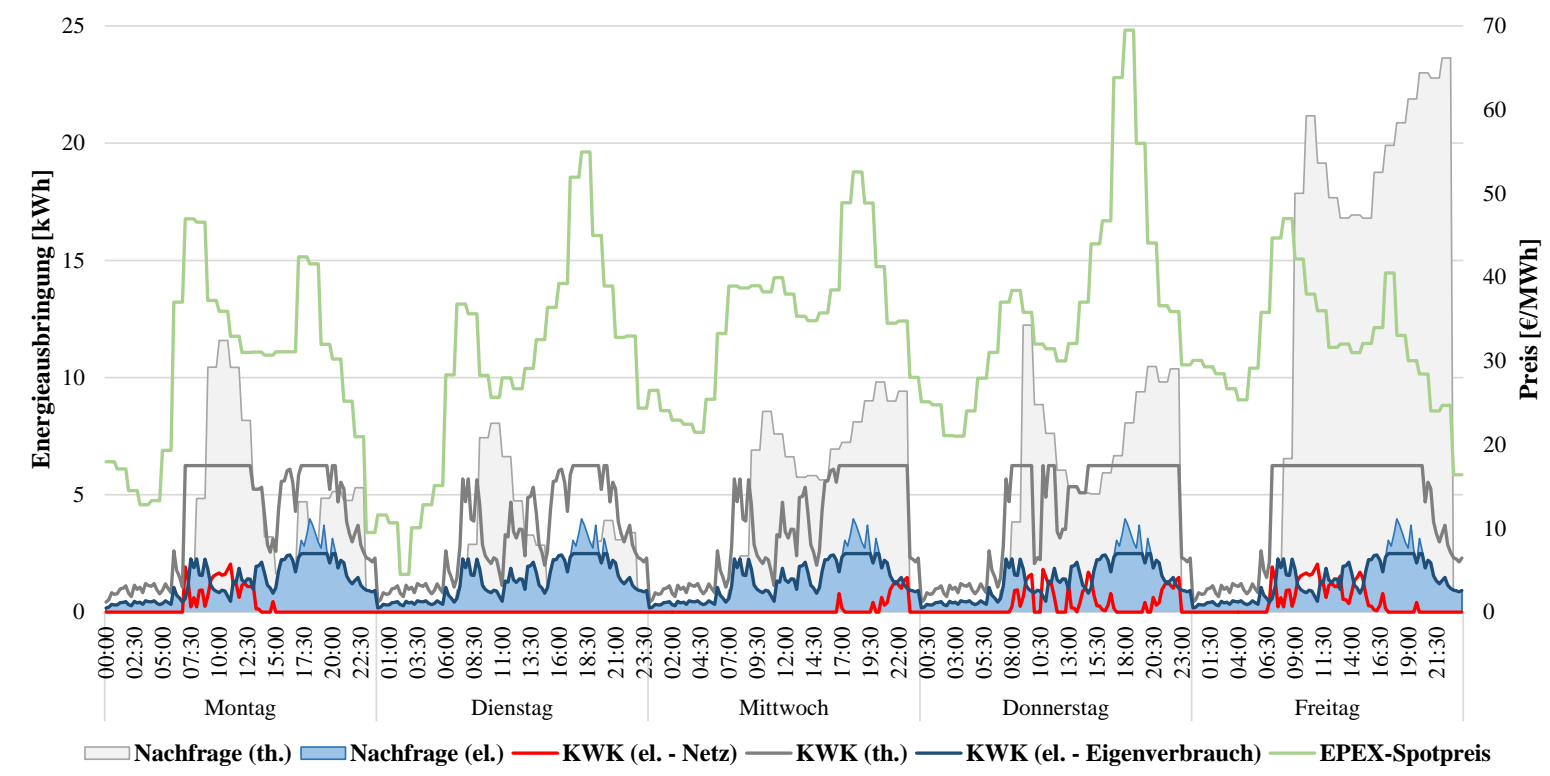

Abb. 11 Verlauf der thermischen und elektrischen Nachfrage sowie der Energieausbringung der KWK-Anlage und der EPEX-Spotpreise für den Fall „EFH_13“ in den Werktagen einer Typwoche im Winter

In der Abbildung ist insbesondere die Fahrweise der KWK-Anlage von Bedeutung. Deren elektrizitätsmarktorientiertes Betriebsverhalten wird in dem betrachteten Zeitraum ersichtlich. Hierzu wird die Energiebereitstellung nach thermischer und elektrischer Energie differenziert und letztere in die Netzeinspeisung und den Eigenverbrauch weiter unterschieden. Zumeist wird die erzeugte Elektrizität in der betrachteten Woche selbst genutzt wie am Abfahren des elektrischen Nachfrageprofils in bestimmten Zeitintervallen zu erkennen ist. Die zeitgleich entstehende Wärme wird im Nichtbedarfsfall in den Wärmespeicher eingespeichert. Die Wärmenachfrage wird zumindest teilweise aus der KWK-Produktion gedeckt. Auftretende Unterdeckungen aus KWK-Wärme werden durch den Spitzenlastkessel bereitgestellt (in Abbildung 11 nicht dargestellt). Weiterhin fördert eine erhöhte thermische Nachfrage wie auch ein gesteigertes Niveau der EPEX-Spotpreise die Einspeisung von Elektrizität in das Netz. An Zeitpunkten des Vorliegens beider Bedingungen wird schließlich die Einspeisung der Elektrizität in das Versorgungsnetz und Erzielen einer marktbasierten Vergütung beobachtet. Hierbei erweist sich auch eine geringere elektrische Nachfrage des Versorgungsobjekts als der Einspeisung zuträglich. 


\subsection{Sensitivitätsanalyse}

Die Ergebnisse der Modellrechnungen hängen in hohem Maße von den verwendeten Eingangsparametern ab. Diese unterscheiden sich unter anderem hinsichtlich des Unsicherheitsgrades der Merkmalsausprägung und in Bezug auf ihre Ergebniseinflussstärke. Eine Sensitivitätsanalyse bietet die Möglichkeit, diese Einflussstärke auf die Ergebnisse durch eine Veränderung der Modellstellgrößen quantitativ aufzuzeigen. Es kann bei diesem Vorgehen jedoch nicht beabsichtigt werden, eine Variation der Annahmen sämtlicher Modellparameter vorzunehmen. Die Analyse muss sich hingegen auf eine begrenzte Auswahl und somit auf die wesentlichen Stellgrößen beschränken. Zudem sollte sich auf solche Merkmale fokussiert werden, deren zukünftige Ausprägungen als besonders unsicher erachtet werden. Im Folgenden werden daher die Höhe der Investition für die KWK-Anlage, die Entwicklung der Endverbraucherpreise für Elektrizität und Erdgas sowie die Entwicklung die Großhandelspreise für Elektrizität (EPEX-Spotpreise, durchschnittliche Quartalspreise) als wesentliche Ergebnisdeterminanten im Untersuchungskontext angesehen. Da selbst mit dieser Beschränkung eine Sensitivitätsanalyse zu allen betrachteten Nachfrageobjekten den Rahmen sprengen würde, wird die Analyse für je einen repräsentativen Fall im mittleren sowie hohen Leistungsbereich, konkret für den Straßenzug mit 13 Einfamilienhäusern sowie die Siedlung mit 204 Einfamilienhäusern, durchgeführt. Die dabei analysierte Ergebnisgröße stellt die relative Abweichung der jeweiligen Zielfunktionswerte, d. h. der Jahresgesamtausgaben für Wärme und Elektrizität unter dem KWKG-Regime 2016 sowie 2012 dar. Weiterhin beträgt die Schrittweite der Abweichung von dem angenommenen Basiswert der untersuchten Modellparameter jeweils $10 \%$-Punkte. Die Abbildung 12 stellt das Ergebnis der Sensitivitätsanalyse graphisch dar.

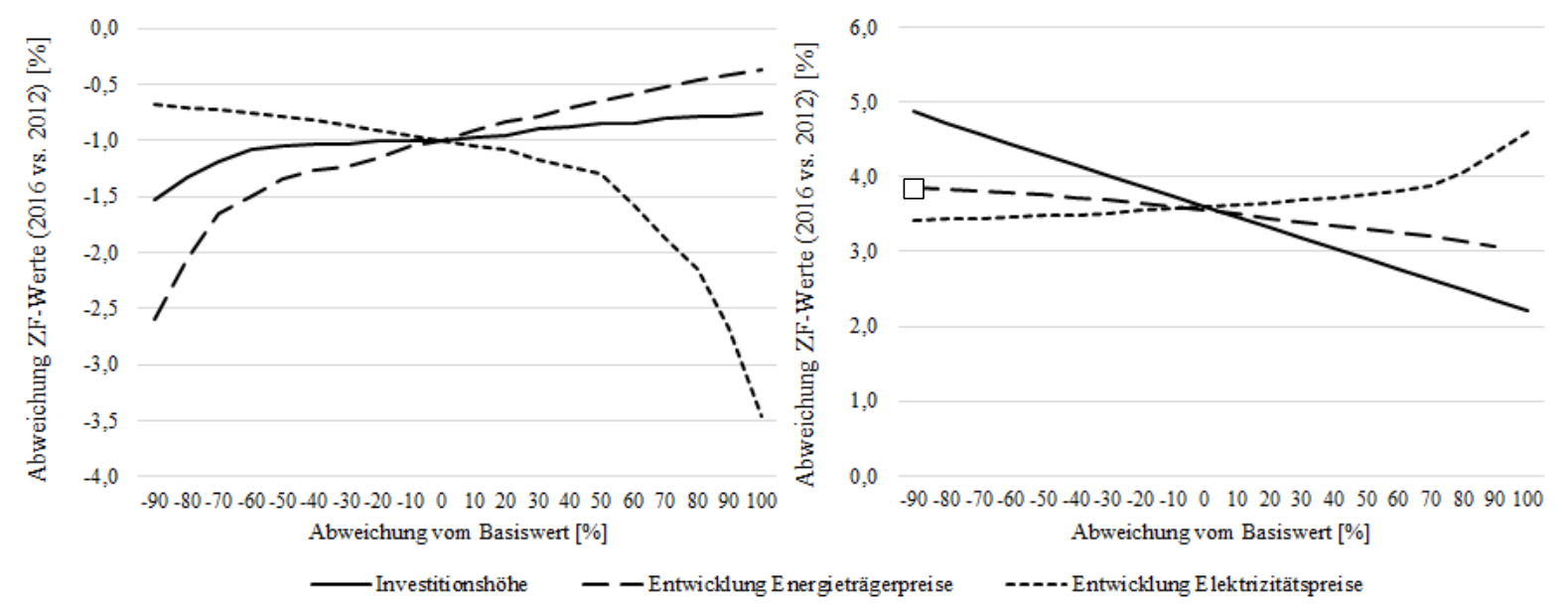

Abb. 12 Sensitivität der prozentualen Abweichung der Zielfunktionswerte bezüglich der Investitionshöhe der KWK-Anlage sowie der Entwicklung der Energieträgerpreise und der Großhandelspreise von Elektrizität für den Fall „EFH_13“ sowie „EFH_204““

Im Ergebnis zeigt sich eine Robustheit der ökonomischen Vorteilhaftigkeit für den KWK-Einsatz durch die Förderung nach dem KWKG 2016 gegenüber der Förderung nach KWKG 2012 für den Fall der 13 Haushalte in den Einfamilienhäusern. Dies wird aus der nicht signifikant vom ursprünglichen Wert um 1,0\% abweichenden Differenz der Zielfunktionswerte für das Intervall der bis zu ca. 50 \% vom Basiswert abweichenden Werte der Eingangsparameter geschlossen. Zudem bleibt über den gesamten untersuchten Bereich die ökonomische Vorteilhaftigkeit des KWK-Einsatzes auf der Fördergrundlage des KWKG 2016 gewahrt. Weiterhin erweist sich 
die analysierte Ergebnisgröße als relativ unempfindlich gegenüber einer Variation der spezifischen Investition. Demgegenüber haben ein geringerer Anstieg der Energieträgerpreise sowie ein höherer Anstieg der Elektrizitätspreise im Großhandel einen stärkeren Ergebniseinfluss und erhöhen die Wirtschaftlichkeit der KWKAnlage unter dem KWKG 2016-Regime.

Für den zweiten untersuchten Anwendungsfall der 204 Einfamilienhäuser wird ebenso eine geringe Sensitivität der relativen Zielfunktionsabweichung unter den beiden KWKG-Regimen vom Basiswert um 3,6 \% konstatiert. Zudem erweist sich die analysierte Ergebnisgröße als relativ insensitiv gegenüber der Entwicklung der Elektrizitätspreise. Somit zeigt sich die ökonomische Vorteilhaftigkeit unter dem Förderregime des KWKG 2012 gegenüber der Nachfolgerversion für den Modellanwendungsfall im hohen Leistungsbereich für die Aggregationsebene von 204 Einfamilienhäusern. ${ }^{6}$ Die Verläufe der Sensitivitätskurven sind dabei als diametral zum Verlauf derjenigen im Anwendungsfall „EFH_13“ zu beschreiben.

Zusammenfassend lässt sich aus der Sensitivitätsanalyse somit eine Robustheit der erzielten Modellergebnisse gegenüber einer Veränderung signifikanter Modelleingangsgrößen im Hinblick auf die ökonomische Vorteilhaftigkeit der Versorgungssysteme unter den beiden Gesetzesfassungen feststellen.

\subsection{Kritische Würdigung des entwickelten Ansatzes}

In diesem Abschnitt wird der entwickelte Modellierungsansatz einer kritischen Würdigung unterzogen.

Ein erster Ansatzpunkt für Kritik bildet die Beschränkung der Analyse auf die Wärmeversorgung von Wohngebäuden sowie auf Verbrennungsmotor-BHKW. Daher kann keine Aussage zu alternativen Technologien und Anwendungsbereichen der Kraft-Wärme-Kopplung getroffen werden. Zusätzliche Anwendungsbereiche wären zum Beispiel im GHD- oder Industriebereich zu identifizieren, welche u. a. durch heterogene Gebäudestrukturen und unterschiedliche Verbrauchsprofile (z. B. geringere Wärmelastschwankungen in der Produktion) gekennzeichnet sind. Ferner bleiben weitere Technologien wie Gasturbinen- oder Organic-RankineCycle-Anlagen sowie Brennstoffzellen-Heizgeräte in der Untersuchung unberücksichtigt. Zudem wird in der Analyse von einer erstmaligen Inbetriebnahme der Technologie ausgegangen, weswegen die weitere Fallunterscheidung im KWKG nach Modernisierung, Nachrüstung sowie Bestandsanlagen vernachlässigt wird. Des Weiteren werden ergänzende, für Wohngebäude relevante (Elektrizitätserzeugungs-)Technologien, wie etwa Photovoltaik und/oder elektrische Speicher, nicht berücksichtigt. Im Rahmen einer tiefergehenden Analyse sollten die technischen und ökonomischen Wechselwirkungen solcher zusätzlichen Technologiekombinationen weiter untersucht werden. Beispielsweise könnte durch einen Batteriespeicher ein zusätzlicher Freiheitsgrad gewonnen werden, um die KWK-Anlage kleiner zu dimensionieren und gleichzeitig die Wirtschaftlichkeit einer Elektrizitätsmarkorientierten Betriebsweise für eine Direktvermarktung zu verbessern. Eine vollständige Untersuchung sämtlicher Einsatzmöglichkeiten von KWK würde jedoch auf Grund der sehr großen Anzahl an Kombinationsmöglichkeiten den Rahmen der Untersuchung übersteigen, weshalb der Untersuchungsrahmen auf eine Technologie sowie einen Sektor beschränkt wurde.

Des Weiteren ist die Annahme der konstanten Ausprägung bestimmter Eingangsparameter kritisch zu hinterfragen. Dies betrifft in erster Linie die Unveränderlichkeit der Lastprofile und Preismuster, welche über den gesamten Planungshorizont von 20 Jahren als konstant angenommen werden. Auch wird der Elektrizitätsmix, der durch die

\footnotetext{
${ }^{6}$ Dies gilt mit Einschränkung einer sehr gemäßigten Entwicklung der Endverbraucherpreise für Elektrizität und Erdgas (10 \% der Referenzentwicklung), bei der sich die Vorteilhaftigkeit umkehrt und die Abweichung auf ca. 21,4 \% beziffert wird. Aus Gründen der Übersichtlichkeit ist dieser Wert jedoch nicht in Abbildung 12 dargestellt.
} 
eingespeiste KWK-Elektrizität verdrängt wird, implizit als unveränderlich angenommen. Demzufolge sind die $\mathrm{CO}_{2}$-Emissionen nur für das Bezugsjahr sinnvoll $\mathrm{zu}$ interpretieren und werden daher für das Jahr 2016 ausgewiesen, für andere Jahre jedoch nicht betrachtet. Zu erwarten ist jedoch, dass sich der Anteil der Stromerzeugung aus $\mathrm{CO}_{2}$-armen Technologien weiter erhöht, so dass die ökologische Vorteilhaftigkeit von KWK reduziert wird. In Wirklichkeit unterliegen auch die Raumwärmebedarfe starken jährlichen und saisonalen Schwankungen und ebenso ist zukünftig ein Rückgang des Raumwärmebedarfs durch verschärfte energiepolitische Verordnungen zum Wärmeschutz zu erwarten. Zudem ist auf Grund der Vielzahl der auf den Preisbildungsprozess einwirkenden unsicheren Parameter von einer Veränderung der Elektrizitätspreise auf dem Großhandelsmarkt gegenüber historischen Verläufen auszugehen. Jedoch ist zumindest die jährliche Veränderlichkeit einer Teilmenge dieser Parameter (Energieträgerpreise sowie EEG-Umlage) durch Berücksichtigung des preisdynamischen Barwertfaktors gegeben. Weiterhin wird in der Sensitivitätsanalyse zumindest ein Teil der Unsicherheit durch Variation wesentlicher Parameter wie die Investition der KWKTechnologien und die Entwicklung der Elektrizitätspreise adressiert.

Die betriebswirtschaftliche Sichtweise des entwickelten und angewandten Modells liefert zwar wertvolle Erkenntnisse für Anlagenbetreiber, lässt aber die volkswirtschaftlichen Effekte außer Acht. Auch aus Gründen des zu begrenzenden Analyseumfangs war es nicht möglich, solche Aspekte zu berücksichtigen. Voraussetzung hierfür wären zum einen eine Erweiterung der Anwendungsfälle über andere Anwendungsbereiche und Leistungsklassen (GHD, Industrie, s. o.) hinweg, zum anderen eine Analyse dieser Anwendungsfälle in einem umfangreichen Energiesystemmodell. Letzteres würde es u. a. ermöglichen, Aussagen zur möglichen Durchdringung von KWKAnlagen im Systemkontext (wie etwa in Merkel (2016), Merkel et al. (2016)) sowie den Wechselwirkungen mit anderen Sektoren zu treffen. Zudem sind nicht sämtliche Einflussgrößen einer betriebswirtschaftlichen Analyse in der durchgeführten wirtschaftlichen Bewertung berücksichtigt. So werden insbesondere Regelungen zur Erhebung bzw. Befreiung von der Umsatzsteuer sowie der Energie- und Stromsteuer in der Betrachtung vernachlässigt. In diesem Zusammenhang könnte überdies die beabsichtigte Änderung des Energiesteuer- und Stromsteuergesetzes bedeutende Auswirkungen auf die Wirtschaftlichkeit der KWK-Systeme in den betrachteten Anwendungsfällen haben (BMF, 2016).

Schließlich wird in der Betrachtung von einem festen Zeithorizont von 20 Jahren ausgegangen. Dabei wird eine gleichmäßige Verteilung der nach KWKG festgelegten gesamten Vollbenutzungsstunden über die Jahre angenommen. Somit wird die Flexibilität der Einsatzplanung eingeschränkt, wodurch zum Beispiel eine Ersatzinvestition in eine KWK-Technologie ausgeschlossen wird. Jedoch wird dieser Aspekt in Anbetracht des Fokus auf den Vergleich der beiden KWKG-Regime und des Vorliegens dieser Problematik in beiden Modellderivaten als nachrangig erachtet, da zumindest für den Ergebnisvergleich von keiner signifikanten Abweichung auszugehen ist.

Zuletzt muss angeführt werden, dass die Versorgung der größeren Aggregationsebenen, insbesondere der Siedlung und des Quartiers, mit einer zentralen KWK-Anlage eine vereinfachte Annahme eines realistischen Betreibermodells darstellt. Ungeachtet der u. a. juristischen Fragestellungen bei der Umsetzung eines solchen Konzepts liegt der Untersuchungsschwerpunkt jedoch auf der technisch-wirtschaftlichen Bewertung der KWKVersorgung auf den verschiedenen Betrachtungsebenen sowie deren Vergleich. Daher werden Aspekte der Machbarkeit in dem vorliegenden Bewertungsansatz vernachlässigt. 


\section{$5 \quad$ Zusammenfassung und Ausblick}

Die vorliegende Arbeit beschäftigt sich mit der Bewertung der Neufassung des Kraft-Wärme-Kopplungsgesetzes aus dem Jahr 2016. Zu diesem Zwecke wird ein bestehender modellgestützter Optimieransatz um die detaillierte Abbildung des KWKG-Vergütungsregimes weiterentwickelt. Der Bewertungsansatz wird im Rahmen der Untersuchung auf acht Anwendungsfälle im Wohngebäudebereich auf unterschiedlichen Aggregationsebenen, von Einzelgebäuden (EFH, MFH) über einen Straßenzug bis hin zu einem Stadtquartier, mit entsprechenden Wärme- und Elektrizitätsnachfragecharakteristika angewendet. Hierbei kommt zur Elektrizitäts-WärmeVersorgung jeweils ein Blockheizkraftwerk zum Einsatz. Im Zentrum der Modellergebnisse steht der quantitative Vergleich des KWKG 2016 und der Vorgängerfassung aus dem Jahr 2012 sowie eines Referenzfalls, in welchem die Wärmeerzeugung in einem Gaskessel und der Elektrizitätsbezug aus dem Netz erfolgt, hinsichtlich Gesamtjahresausgaben und induzierten Einzahlungen, Elektrizitätsnetzinteraktion, Energieautarkie sowie $\mathrm{CO}_{2}$ Emissionen.

Die Modellergebnisse zeigen für das Bezugsjahr 2016, dass eine ökonomische Vorteilhaftigkeit unter den Regelungen des KWKG 2016 gegenüber der Vorgängerversion in dem mittleren Leistungsbereich des Straßenzugs und Häuserblocks, d.h. für 13 und 100 Haushalte, gegeben ist. Im Gegensatz dazu wird die Wirtschaftlichkeit unter den Förderbedingungen des KWKG 2016 bei den größten Objekten mit 204 und 1.000 Haushalten (Siedlung, Quartier) verschlechtert. Beide Sachverhalte sind auf die gegenläufige Abhängigkeit der Zuschlagsdauer und der Anlagenkapazität im KWKG 2016 zurückzuführen. So wird bei den vier Anwendungsfällen der größten Aggregationsebenen eine Leistung von $50 \quad \mathrm{~kW}_{\mathrm{el}}$ installiert, $\mathrm{zu}$ welcher die bezuschlagte Vollbenutzungsstundenanzahl gegenüber höheren Leistungen verdoppelt ist. Ferner wird bei allen sechs Anwendungsfällen mit KWK-Installation die erhöhte Anzahl der Vollbenutzungsstunden von 3.000 Stunden im Jahr bei einem Betrachtungshorizont von 20 Jahren ausgenutzt. Die Umkehr der Wirtschaftlichkeit bei den Anwendungsfällen des höheren Verbrauchs (wie etwa von 204 und 1.000 Haushalten) liegt darin begründet, dass keine weiteren Größendegressionseffekte in Anspruch genommen werden können, weswegen die Jahresgesamtausgaben unter den Regelungen des KWKG 2016 höher sind. Bei den zwei Anwendungsfällen in einzelnen Einfamilienhäusern werden hingegen unter beiden Fördersystemen keine KWK-Anlagen installiert, was der relativ geringen Gesamtwärmenachfrage in diesen Objekten geschuldet ist.

Die Anwendungsfälle mit 100, 204 und 1000 Haushalten sind insofern Konfliktfälle, als dass eine Abwägung zwischen Anlagenkapazität und Volllaststunden vorherrscht. Im Prinzip ist die Gesamtwärmenachfrage in diesen Objekten hoch genug, um eine größere Auslegung als die gewählte Kapazität von 50 kW el wenigstens technisch zu begründen, aber dafür würden die Anlagen den gesetzlichen Zuschlag nur für die Hälfte der Volllaststunden in Anspruch nehmen können. Mit der Ausnahme von dem Fall von 100 Haushalten in einem MFH reduziert sich deswegen unter dem KWKG 2016 der Autarkiegrad und die Netzinteraktion. Zudem erhöhen sich der Eigenverbrauchsanteil und somit auch der Anteil der zuschlagsfähigen Elektrizitätserzeugung in diesen Fällen zwischen den Gesetzesversionen. Für die Fälle mit 13 Haushalten verhält sich diese Tendenz umgekehrt, werden also höhere Autarkiegrade und Netzinteraktion, niedrigere Eigenverbrauchsanteile und Anteile der zuschlagsfähigen Elektrizitätsgestehung ermittelt. Diese Verhältnisse spiegeln sich auch in den Ergebnissen zu den $\mathrm{CO}_{2}$-Emissionen wider, d. h. die vermiedenen Emissionen hängen in hohem Maße von der installierten Leistung und der Menge der eingespeisten Elektrizität ab. 
Der vorliegende Beitrag liefert somit Erkenntnisse zur Effektivität der KWK-Novelle 2016 für Wohngebäude aus einzelwirtschaftlicher Sicht. Allerdings sind einige Aspekte der entwickelten und angewendeten Methodik durchaus ausbaufähig. Der Fokus der Untersuchung ist auf Wohngebäude und Verbrennungsmotoren gerichtet. So werden höhere Leistungsbereiche, andere zukunftsorientierte bzw. ergänzende Technologien wie Brennstoffzellen bzw. Photovoltaik und Batteriespeicher sowie andere Anwendungsbereiche in der Industrie oder im GHD-Sektor von der Betrachtung ausgeschlossen. Der „grüne Wiese“-Ansatz ermöglicht es zudem nicht, eine Unterscheidung zwischen Modernisierung, Nachrüstung und Bestandsanlagen vorzunehmen. Dazu unterliegt eine Vielzahl der Modelleingangsparameter, die notwendigerweise weit in die Zukunft fortgeschrieben werden müssen, großen Unsicherheiten. Wie im Rahmen einer Sensitivitätsanalyse untersucht, beeinflussen diese die relevanten Ergebnisse des Vergleichs der Förderregime der KWKG-Versionen jedoch nur geringfügig. Schließlich bleiben auch die volkswirtschaftlichen Auswirkungen des erneuerten gesetzlichen Rahmens unberücksichtigt. Die Voraussetzungen hierfür wären u. a. eine erhebliche Erweiterung des Modellumfangs, wie dargelegt, um andere KWK-Technologien, Leistungsbereiche und Anwendungsbereiche, sowie eine detaillierte Abbildung anderer Sektoren in einem ganzheitlichen Energiesystemmodell. 


\begin{tabular}{ll}
\hline Abkürzung & Beschreibung \\
\hline & \\
BHKW & Blockheizkraftwerk \\
EEG & Erneuerbare Energien-Gesetz \\
EEX & European Energy Exchange \\
EFH & Einfamilienhaus \\
EG & Energieautarkiegrad \\
EL & Elektrizität \\
EPEX & European Power Exchange \\
EV & Eigenverbrauch \\
GAMS & General Algebraic Modeling System \\
GASK & Gaskessel \\
GHD & Gewerbe, Handel, Dienstleistungen \\
HH & Haushalte \\
Kap & Kapazität \\
KWK & Kraft-Wärme-Kopplung \\
KWKG & Kraft-Wärme-Kopplungsgesetz \\
MFH & Mehrfamilienhaus \\
mKWK & mikro-Kraft-Wärme-Kopplung \\
NII & Netzinteraktionsindex \\
SOS2 & Special Ordered Sets of type 2 \\
VBSt & Vollbenutzungsstunden \\
Vol & Volumen \\
WSP & Wärmespeicher \\
&
\end{tabular}

Tab. A.1 Symbole und Beschreibung

\begin{tabular}{|c|c|c|c|}
\hline Symbol & Beschreibung & Einheit & Wert/Elemente \\
\hline$\propto$ & Gewichtungsfaktor der zeitlichen Dekomposition & - & - \\
\hline$c_{\text {fix, } p}$ & Fixe betriebsgebundene jährliche Ausgaben von $\mathrm{p}$ & $€$ & - \\
\hline$c_{\text {inv, } p}$ & Kapitalgebundene jährliche Ausgaben von p & $€$ & - \\
\hline $\mathrm{c}_{\mathrm{rev}, \mathrm{e}}\left(\mathrm{x}_{\mathrm{cap}}^{\mathrm{KWK}}\right)$ & $\begin{array}{l}\text { Spezifische Einzahlungen für erzeugte und } \\
\text { eingespeiste Elektrizität der KWK-Anlage in } \\
\text { Abhängigkeit von } \mathrm{x}_{\mathrm{cap}}^{\mathrm{KWK}}\end{array}$ & $€ / \mathrm{kWh}_{\mathrm{el}}$ & - \\
\hline $\mathrm{c}_{\mathrm{rev}, \mathrm{i}}\left(\mathrm{x}_{\mathrm{cap}}^{\mathrm{KWK}}\right)$ & $\begin{array}{l}\text { Spezifische Einzahlungen für erzeugte und } \\
\text { selbstgenutzte Elektrizität der KWK-Anlage in } \\
\text { Abhängigkeit von } \mathrm{x}_{\text {cap }}^{\mathrm{KWK}}\end{array}$ & $€ / \mathrm{kWh}_{\mathrm{el}}$ & - \\
\hline $\mathrm{c}_{\mathrm{rev}, \mathrm{p}, \mathrm{t}}$ & Einzahlungen von $\mathrm{p}$ in $\mathrm{t}$ & $€$ & - \\
\hline$c_{\mathrm{var}, \mathrm{p}, \mathrm{t}}$ & Verbrauchsgebundene Ausgaben von $\mathrm{p}$ in $\mathrm{t}$ & $€$ & - \\
\hline $\operatorname{dem}_{e l}(t)$ & Nachfrage nach Elektrizität in $\mathrm{t}$ & $\mathrm{kWh}_{\mathrm{el}}$ & - \\
\hline$\varepsilon$ & Kleine Zahl & - & 0,01 \\
\hline $\mathrm{E}(\mathrm{t})$ & Netto-Elektrizitätsexport in $\mathrm{t}$ & $\mathrm{kWh}_{\mathrm{el}}$ & - \\
\hline$f$ & Zielfunktionswert & $€$ & - \\
\hline $\mathrm{i}$ & Zinssatz & $\%$ p.a. & 5 \\
\hline $\mathrm{p}$ & Index für Technologien & - & - \\
\hline $\mathrm{P}$ & Menge der Technologien & - & $\{\mathrm{EL} ; \mathrm{GASK} ; \mathrm{KWK} ; \mathrm{WSP}\}$ \\
\hline $\mathrm{t}$ & Index für Zeitschritt & - & - \\
\hline $\mathrm{T}$ & Menge der Zeitschritte & - & $\{1 ; \ldots 6048\}$ \\
\hline $\mathrm{t}^{\prime}$ & Index für Modelljahre & - & - \\
\hline $\mathrm{T}^{\prime}$ & Planungszeitraum & $\mathrm{a}$ & 20 \\
\hline $\mathrm{x}_{\mathrm{cap}}^{\mathrm{KWK}}$ & Installierte Kapazität der KWK-Anlage & $\mathrm{kW}_{\mathrm{el}}$ & - \\
\hline$x_{e l, e}^{K W K}(t)$ & $\begin{array}{l}\text { Erzeugte und eingespeiste Elektrizität der KWK- } \\
\text { Anlage in } \mathrm{t}\end{array}$ & $\mathrm{kWh}_{\mathrm{el}}$ & - \\
\hline
\end{tabular}




\begin{tabular}{|l|l|l|l|}
\hline Symbol & Beschreibung & Einheit & Wert/Elemente \\
\hline$x_{e l, i}^{K W K}(t)$ & $\begin{array}{l}\text { Erzeugte und selbstgenutzte Elektrizität der KWK- } \\
\text { Anlage in t }\end{array}$ & $\mathrm{kWh}_{\mathrm{el}}$ & - \\
\hline $\mathrm{y}_{K W K, 1}^{E E G}$ & $\begin{array}{l}\text { Binärvariable zur Befreiung von der anteiligen } \\
\text { EEG-Umlage }\end{array}$ & - & $\{0 ; 1\}$ \\
\hline $\mathrm{y}_{K W K, 2}^{E E G}$ & $\begin{array}{l}\text { Binärvariable zur Entrichtungspflicht der } \\
\text { anteiligen EEG-Umlage }\end{array}$ & - & $\{0 ; 1\}$ \\
\hline
\end{tabular}

\section{Anhang B. Numerische Annahmen}

Tab. B.1 Technische und wirtschaftliche Charakteristika der Technologien (Quelle: ASUE (2014), VDI (2012), eigene Annahmen)

\begin{tabular}{|l|l|l|l|l|l|}
\hline Technologie & \multicolumn{2}{|l|}{$\begin{array}{l}\text { Betriebsgebundene jahresfixe } \\
\text { Ausgaben }\end{array}$} & Jahresnutzungsgrad & Nutzungsdauer & Stromkennzahl \\
\hline & {$\left[€ /\left(\mathrm{kW}_{\mathrm{el}} \mathrm{a}\right)\right]$} & {$\left[€ /\left(\mathrm{kW}_{\mathrm{th}} \mathrm{a}\right)\right]$} & - & $\mathrm{a}$ & - \\
\hline Gaskessel & - & 25,9 & 0,9 & 20 & - \\
\hline BHKW & 150 & - & 0,9 & 20 & 0,4 \\
\hline
\end{tabular}

Tab. B.2 Primärenergie- und $\mathrm{CO}_{2}$-Emissionsfaktoren (Quelle: eigene Berechnung auf Basis von (Fritsche et al. 2014))

\begin{tabular}{|l|l|l|}
\hline Energieträger & Primärenergiefaktor (gesamt) $[\mathrm{TJ} / \mathrm{TJ}]$ & $\mathrm{CO}_{2}$-Emissionsfaktor (dir+indir) $\left[\mathrm{t}_{\mathrm{CO} 2} / \mathrm{TJ}_{\mathrm{Hi}}\right]$ \\
\hline Erdgas & 1,128 & 66,260 \\
\hline Elektrizität & 2,6 & 138,89 \\
\hline
\end{tabular}




\section{Literatur}

ASUE (2014) BHKW-Kenndaten 2014/2015. Arbeitsgemeinschaft für sparsamen und umweltfreundlichen Energieverbrauch e.V., Berlin.

ASUE et al. (2015) Neues KWKG 2016 für eine erfolgreiche Energiewende im Strom- und Wärmemarkt nutzen. Gemeinsames Verbändepapier zum Entwurf für die Neufassung des KWKG. Arbeitsgemeinschaft für sparsamen und umweltfreundlichen Energieverbrauch e.V., Berlin.

BAFA (2016) KWK-Anlagen - Bestand in Deutschland nach Größenklasse 2015. http://de.statista.com/statistik/daten/studie/468203/umfrage/anzahl-der-kwk-anlagen-in-deutschland/.

Zugegriffen: 31. Mrz. 2016

Bardt H, Chrischilles E, Growitsch C, Hagspiel S, Schaupp L (2014) Eigenerzeugung und Selbstverbrauch von Strom - Stand, Potenziale und Trends. Z Energiewirtschaft 38:83-99

BMF (2016) Entwurf eines Zweiten Gesetzes zur Änderung des Energiesteuer- und des Stromsteuergesetzes. http://www.bundesfinanzministerium.de/Content/DE/Gesetzestexte/Referentenentwuerfe/2016-05-19Energiesteuer-Download.pdf? blob=publicationFile\&v=2. Zugegriffen: 01. Jun. 2016

BMWi (2016) Zahlen und Fakten Energiedaten. Nationale und Internationale Entwicklung. Bundesministerium für Wirtschaft und Energie. http://www.bmwi.de/DE/Themen/Energie/Energiedaten-undanalysen/Energiedaten/gesamtausgabe,did=476134.html. Zugegriffen: 30. Mrz. 2016

Fritsche U R, Rausch L, Schmidt K (2014) Dokumentation zur Aktualisierung und Ergänzung der GEMIS-Daten in Version 4.5. Öko-Institut e.V., Darmstadt.

Heffels T, McKenna R, Fichtner W (2012) Direct marketing of electricity from biogas and biomethane: an economic analysis of several business models in Germany. Journal of Management Control 23:53-70. Doi: $10.1007 / \mathrm{s} 00187-012-0153-\mathrm{Z}$

McKenna R, Herbes C, Fichtner W (2015) Energieautarkie: Vorschlag einer Arbeitsdefinition als Grundlage für die Bewertung konkreter Projekte und Szenarien. Z Energiewirtschaft 39:235-252

McKenna R, Merkel E, Fichtner W (2016) Energy autonomy in residential buildings: A techno-economic modelbased analysis of the scale effects. Applied Energy, (in press) Doi:10.1016/j.apenergy.2016.03.062

Merkel E, McKenna R, Fichtner W (2015) Optimisation of the capacity and the dispatch of decentralised microCHP systems: a case study for the UK. Applied Energy 140:120-134

Merkel E, McKenna R, Fehrenbach D, Fichtner W (2016) A model-based assessment of climate and energy targets for the German residential heat system. Journal of Cleaner Production (submitted)

Merkel E (2016) Analyse und Bewertung des Elektrizitätssystems und des Wärmesystems der Wohngebäude in Deutschland. Dissertation, Karlsruher Institut für Technologie.

Salom J, Widén J, Candanedo J, Sartori I, Voss K, Marszal A (2011) Understanding net zero energy buildings: evaluation of load matching and grid interaction indicators. Proceedings of building simulation 6:2514-2521

Schlesinger M, Lindenberger D, Lutz C (2014) Entwicklung der Energiemärkte - Energiereferenzprognose. Studie im Auftrag des Bundesministeriums für Wirtschaft und Technologie, Berlin.

VDI (2012) Wirtschaftlichkeit gebäudetechnischer Anlagen - Grundlagen und Kostenberechnung, VDI-Richtlinie 2067. Verein Deutscher Ingenieure e.V., Düsseldorf. 


\section{Working Paper Series in Production and Energy}

recent issues

No. 1 Alexandra-Gwyn Paetz, Lisa Landzettel, Patrick Jochem, Wolf Fichtner: Eine netnografische Analyse der Nutzererfahrungen mit E-Rollern

No. 2 Felix Teufel, Michael Miller, Massimo Genoese, Wolf Fichtner: Review of System Dynamics models for electricity market simulations

No. 3 Patrick Jochem, Thomas Kaschub, Wolf Fichtner:

How to integrate electric vehicles in the future energy system?

No. 4 Sven Killinger, Kai Mainzer, Russell McKenna, Niklas Kreifels and Wolf Fichtner: A regional simulation and optimisation of renewable energy supply from wind and photovoltaics with respect to three key energy-political objectives

No. 5 Kathrin Dudenhöffer, Rahul Arora, Alizée Diverrez, Axel Ensslen, Patrick Jochem, Jasmin Tücking: Potentials for Electric Vehicles in France, Germany, and India

No. 6 Russell McKenna, Carsten Herbes, Wolf Fichtner: Energieautarkie: Definitionen, Für- bzw. Gegenargumente, und entstehende Forschungsbedarfe

No. 7 Tobias Jäger, Russell McKenna, Wolf Fichtner: Onshore wind energy in Baden- Württemberg: a bottom-up economic assessment of the socio-technical potentia

No. 8 Axel Ensslen, Alexandra-Gwyn Paetz, Sonja Babrowski, Patrick Jochem, Wolf Fichtner: On the road to an electric mobility mass market - How can early adopters be characterized?

No. 9 Kai Mainzer, Russell McKenna, Wolf Fichtner:

Charakterisierung der verwendeten Modellansätze im Wettbewerb Energieeffiziente Stadt

No. 10

Hannes Schwarz, Valentin Bertsch, Wolf Fichtner: Two-stage stochastic, large-scale optimization of a decentralized energy system - a residential quarter as case study

No. 11 Leon Hofmann, Russell McKenna, Wolf Fichtner: Development of a multi-energy residential service demand model for evaluation of prosumers' effects on current and future residential load profiles for heat and electricity

No. 12 Russell McKenna, Erik Merkel, Wolf Fichtner: Energy autonomy in residential buildings: a techno-economic model-based analysis of the scale effects

No. 13 Johannes Schäuble, Silvia Balaban, Peter Krasselt, Patrick Jochem, Mahmut Özkan, Friederike Schellhas-Mende, Wolf Fichtner, Thomas Leibfried, Oliver Raabe: Vergleichsstudie von Systemansätzen für das Schnellladen von Elektrofahrzeugen

The responsibility for the contents of the working papers rests with the author, not the institute. Since working papers are of preliminary nature, it may be useful to contact the author of a particular working paper about results or caveats before referring to, or quoting, a paper. Any comments on working papers should be sent directly to the author. 


\section{Working Paper Series in Production and Energy}

recent issues

No. 14 Marian Hayn, Valentin Bertsch, Anne Zander, Stefan Nickel, Wolf Fichtner: The impact of electricity tariffs on residential demand side flexibility

No. 15 Erik Merkel, Robert Kunze, Russell McKenna, Wolf Fichtner: Modellgestützte Bewertung des Kraft-Wärme-Kopplungsgesetzes 2016 anhand ausgewählter Anwendungsfälle in Wohngebäuden

The responsibility for the contents of the working papers rests with the author, not the institute. Since working papers are of preliminary nature, it may be useful to contact the author of a particular working paper about results or caveats before referring to, or quoting, a paper. Any comments on working papers should be sent directly to the author. 


\section{Impressum}

Karlsruher Institut für Technologie

Institut für Industriebetriebslehre und Industrielle Produktion (IIP) Deutsch-Französisches Institut für Umweltforschung (DFIU)

Hertzstr. 16

D-76187 Karlsruhe

KIT - Universität des Landes Baden-Württemberg und

nationales Forschungszentrum in der Helmholtz-Gemeinschaft

Working Paper Series in Production and Energy

No. 15, Juni 2016

ISSN 2196-7296

www.iip.kit.edu 